\title{
Shape Optimization for Delay of Laminar-Turbulent Transition
}

\author{
Olivier Amoignon* \\ Uppsala University, SE-751 05 Uppsala, Sweden \\ Jan Pralits ${ }^{\dagger}$ \\ Università di Salerno, 84084 Fisciano (SA), Italy \\ and \\ Ardeshir Hanifi, ${ }^{\ddagger}$ Martin Berggren, ${ }^{\S}$ and Dan Henningson ${ }^{\text {II }}$ \\ Swedish Defence Research Agency, SE-16490 Stockholm, Sweden
}

\begin{abstract}
A method using gradient-based optimization is introduced for the design of wing profiles with the aim of natural laminar flow, as well as minimum wave drag. The Euler equations of gasdynamics, the laminar boundary-layer equations for compressible flows on infinite swept wings, and the linear parabolized stability equations (PSE) are solved to analyze the evolution of convectively unstable disturbances. Laminar-turbulent transition is assumed to be delayed by minimizing a measure of the disturbance kinetic energy of a chosen disturbance, which is computed using the PSE. The shape gradients of the disturbance kinetic energy are computed based on the solutions of the adjoints of the state equations just named. Numerical tests are carried out to optimize the RAE 2822 airfoil with the aim to delay simultaneously the transition, reduce the pressure drag coefficient, and maintain the coefficients of lift and pitch moments. Constraints are also applied on the geometry. Results show a reduction of the total amplification of a large number of disturbances, which is assumed to represent a delay of the transition in the boundary layer. Because delay of the transition implies reduction of the viscous drag, the present method enables shape optimization to perform viscous drag reduction.
\end{abstract}

\begin{tabular}{ll} 
& \multicolumn{1}{c}{ Nomenclature } \\
$A_{s}$ & $=$ stiffness matrix for $\mathrm{d}^{2} / \mathrm{d} s^{2}$ \\
$\boldsymbol{a}$ & $=$ design variables, $\mathbb{R}^{n}$ \\
$\boldsymbol{b}$ & $=$ values of geometrical constraints, $\mathbb{R}^{m}$ \\
$C$ & $=$ constant coefficients matrix, $\mathbb{R}^{n \times m}$ \\
$C_{D}, C_{L}, C_{M}$ & $=$ drag, lift, and pitch-moment coefficients \\
$C_{p}$ & $=$ pressure coefficient \\
$c$ & $=$ sound speed, $\mathrm{m} / \mathrm{s}$ \\
$c_{p}$ & $=$ specific heat at constant pressure \\
$d$ & $=$ dimension, 2 \\
$E$ & $=$ total energy density, $\mathrm{kg} / \mathrm{m} / \mathrm{s}^{2}$ \\
$\mathcal{E}$ & $=$ set of edges \\
$\boldsymbol{f}$ & $=$ flux density, 3 -by-1 matrix of tensors \\
$h_{1}$ & $=$ scale factor, curvilinear coordinates \\
$i j$ & $=$ edge between nodes $i$ and $j$ \\
$L$ & $=$ constant coefficients matrix, $\mathbb{R}^{d N \times n}$ \\
$l_{0} \times$ reference length scale, $\mathrm{m}$ \\
$M$ & $=$ Mach number \\
$M_{Q}$ & $=$ diagonal matrix diag $(0,1,1,1,0)$ \\
$M_{s}$ & $=$ mass matrix \\
$m$ & $=$ number of geometric constraints \\
$\boldsymbol{m}$ & $=$ momentum density $\rho \boldsymbol{u}$ \\
$N$ & $=$ total number of nodes in Euler grid \\
$N_{E}$ & $=N$ factor based on disturbance kinetic energy
\end{tabular}

Received 29 July 2004; revision received 10 October 2005; accepted for publication 13 November 2005. Copyright (C) 2005 by the Swedish Defence Research Agency. Published by the American Institute of Aeronautics and Astronautics, Inc., with permission. Copies of this paper may be made for personal or internal use, on condition that the copier pay the $\$ 10.00$ per-copy fee to the Copyright Clearance Center, Inc., 222 Rosewood Drive, Danvers, MA 01923; include the code 0001-1452/06 \$10.00 in correspondence with the CCC.

*Ph.D. Student, Department of Information Technology.

${ }^{\dagger}$ Researcher, Department of Mechanical Engineering; also Research Engineer, Swedish Defence Research Agency, SE-164 90 Stockholm, Sweden. Member AIAA.

${ }^{\ddagger}$ Researcher, Aeronautics Division

${ }^{\S}$ Researcher, Aeronautics Division; also Senior Lecturer, Department of Information Technology, Uppsala University, SE-751 05 Uppsala, Sweden.

IIResearcher, Aeronautics Division; also Professor, Department of Mechanics, Royal Institute of Technology, SE-100 44 Stockholm, Sweden.

\begin{tabular}{|c|c|}
\hline $\mathcal{N}_{i}$ & $=$ set of nodes connected to $i$ by edge \\
\hline$n$ & $=$ number of nodes on airfoil \\
\hline$n$ & $=$ outward directed surface normal vector \\
\hline$\hat{\boldsymbol{n}}$ & outward directed unit normal vector \\
\hline$P$ & $=$ pressure, dimensionless \\
\hline $\operatorname{Pr}$ & $=$ Prandtl number \\
\hline$p$ & $=$ pressure, $\mathrm{Pa}$ \\
\hline $\bar{Q}$ & $=$ state of mean flow, $[\bar{U}, \bar{V}, \bar{W}, \bar{T}]_{\sim}^{T} \sim$ \\
\hline$\widetilde{\tilde{Q}}$ & $=$ disturbance state vector, $[\tilde{\rho}, \tilde{U}, \tilde{\tilde{V}}, \tilde{W}, \tilde{T}]^{T}$ \\
\hline$\widetilde{\mathcal{R}}$ & $=$ specific gas constant \\
\hline$R e_{s}, R e$ & $=$ reynolds numbers based on lengths $s$ and $l_{0}$ \\
\hline $\boldsymbol{R}_{i}$ & $=$ residual at node $i, 3 \times 1$ matrix of tensors \\
\hline$s_{s}$ & $=$ arclength from stagnation point \\
\hline$s_{t}$ & $=$ arclength from trailing edge \\
\hline$T$ & $=$ temperature, dimensionless \\
\hline$t$ & $=$ time \\
\hline$U, V, W$ & $=$ velocity components, dimensionless \\
\hline $\boldsymbol{u}$ & $=$ velocity tensor, $\mathrm{m} / \mathrm{s}$ \\
\hline$V$ & $=$ dual control volume \\
\hline $\mathcal{V}(\Omega)$ & $=$ set of nodes in $\Omega$ \\
\hline $\mathcal{V}(\partial \Omega)$ & $=$ set of boundary nodes of $\Omega$ \\
\hline$v$ & $=$ primitive variables, $[\rho, \boldsymbol{u}, p]^{T}$ \\
\hline$w$ & $=$ conservative variables, $[\rho, \boldsymbol{m}, E]^{T}$ \\
\hline $\boldsymbol{X}$ & $=$ vector of Euler nodal coordinates, $\mathbb{R}^{N d}$ \\
\hline$X_{n 1}$ & $=$ branch 1 of neutral stability curve \\
\hline$X_{S}$ & $=$ streamwise position of stagnation point \\
\hline$X_{1}$ & $=$ downstream position of viscous computation \\
\hline$x, y, z$ & $=$ cartesian body axes, $\mathrm{m}$ \\
\hline$x^{1}, x^{2}, x^{3}$ & $=$ curvilinear coordinates \\
\hline$y$ & $=$ nodal displacements on the airfoil, $\mathbb{R}^{n}$ \\
\hline$Z_{0}, Z_{1}$ & $=$ spanwise periodic interval boundaries \\
\hline$\alpha, \beta$ & $=$ streamwise, spanwise wave number \\
\hline$\alpha_{l}$ & $=$ angle of attack, deg \\
\hline$\gamma$ & $=$ ratio of specific heats \\
\hline$\eta$ & $=$ wall-normal distance, $\mathrm{m}$ \\
\hline$\kappa$ & $=$ heat conductivity, dimensionless \\
\hline$\lambda$ & $=$ vector of Lagrange multipliers, $\mathbb{R}^{m}$ \\
\hline$\mu, v$ & $=$ dynamic, kinematic viscosities, dimensionless \\
\hline$\rho$ & $=$ density, $\mathrm{kg} / \mathrm{m}^{3}$ \\
\hline$\sigma$ & $=$ growth rate \\
\hline$\psi$ & $=$ sweep angle \\
\hline
\end{tabular}




$$
\begin{array}{ll}
\Omega & =\text { domain of discretized Euler equations } \\
\Omega_{B}, \Omega_{P} & =\text { domains for mean flow and stability analysis } \\
\omega & =\text { angular frequency }
\end{array}
$$

\section{Subscripts}

$\begin{array}{ll}e & =\text { boundary-layer edge } \\ i & =\text { node index } \\ i j & =\text { edge index } \\ w & =\text { wall } \\ 0 & =\text { reference position } \\ \infty & =\text { freestream }\end{array}$

\section{Superscripts}

$\begin{array}{ll}H & =\text { conjugate transpose } \\ k & =k \text { th design } \\ n+1 & =\text { time level } \\ T & =\text { transpose } \\ 0 & =\text { initial design } \\ * & =\text { adjoint quantity } \\ - & =\text { dimensionless mean flow quantity } \\ \sim & =\text { amplitude function } \\ & =\text { dimensionless disturbance quantity }\end{array}$

\section{Introduction}

D RAG reduction for high-speed vehicles is a challenging task. The use of laminar flow control techniques aims at delaying the laminar-turbulent transition, which is known to reduce the viscous drag coefficient. The research in the area regarding active flow control is vast; see Joslin ${ }^{1}$ for a thorough review on the topic of laminar flow control. Computational fluid dynamics (CFD)-based design optimization has proved to be successful in reducing the pressure drag at transonic flow regime; see, for example, Jameson. ${ }^{2}$ However, attempts to minimize the total drag by regular CFD-based design optimization have relied on a fixed laminar-turbulent transition point or on the assumption that the flow is fully turbulent, as in Ref. 3.

Laminar-turbulent transition in the boundary layer on aircraft wings is usually caused by the breakdown of small disturbances that grow as they propagate downstream. The amplification of these disturbances can be analyzed using linear stability theory, in which it is assumed that disturbances with infinitesimal amplitude are superimposed on the laminar mean flow. The growth rate of the disturbances can then be used to predict the transition location using the so-called $e^{N}$ method. ${ }^{4-6}$ Linear stability analysis has been used in some investigations with the aim of designing shapes such that the laminar portion is increased. This approach is denoted as natural laminar flow (NLF) design. In Ref. 7, a target pressure is found, based on a simplified relation between pressure and $N$ factor, which is used to state an inverse problem to find a geometry that may delay transition. In Ref. 8, a surface panel method was coupled with an approximative boundary-layer calculation and linear stability analysis. However, none of these investigations calculated the sensitivities based on the linear stability analysis to formulate optimality conditions.

Both CFD-based shape optimization and flow control approaches may be treated as optimization problems constrained by systems of partial differential equations (PDEs). When an adjoint system of partial differential equations is solved, the gradient of the objective function can be obtained at a cost that is independent of the number of parameters subject to the optimization. The use of adjoint equations in design optimization may be viewed as an offspring of the theory of optimal control for PDE developed by Lions. ${ }^{9}$ Based on this approach, the optimal shape of a body in Stokes flows was derived by Pironneau ${ }^{10}$ in 1973. In 1988, Jameson ${ }^{11}$ formulated the adjoints of the full potential flow equations and of the Euler equations to solve inverse problems. Thereafter, research teams have developed adjoint codes for the design of aircrafts with lower drag. ${ }^{12-21}$ The reader will find an introduction to the method of adjoints applied to aerodynamic design in Ref. 22. As mentioned earlier, an approach of optimal NLF design is to formulate an inverse problem in which the target pressure is designed to damp the amplification of disturbances and the parameters of optimization describe the shape, as in Refs. 23-25. Inverse problems of this kind were investigated first by Lighthill ${ }^{26}$ and, through an optimal control approach, by Jameson. ${ }^{11}$

In the present contribution, a CFD calculation is coupled to an analysis of the growth rate of disturbances superimposed on the laminar flow in the boundary layer. With this approach, the shape, here an airfoil, can be optimized with respect to the energy of disturbances for the purpose of delaying laminar-turbulent transition. To relate the amplification of disturbances to the parameterization of the shape, and to keep computational costs at a reasonable level, the flow is divided into an inviscid outer flowfield and a viscous part describing the boundary layer at the surface. In this way, the growth rate of a disturbance superimposed on the boundary layer of a given geometry (shape) can be calculated as follows:

1) The solution of the Euler equations provides a pressure distribution on the surface of the geometry defined by the design parameters.

2) The viscous mean flow is obtained by solving the boundarylayer equations for compressible flows over infinite swept wings, given the pressure distribution and the geometry.

3) The linear stability equations are solved given the viscous mean flow and the geometry, providing the amplitude and phase of a specific disturbance.

It has been shown by Pralits and Hanifii ${ }^{27}$ and Pralits et al. ${ }^{28}$ that reducing a measure of the kinetic energy of a specific disturbance causes a reduction of the total amplification of a large number of disturbances, which can be assumed to represent a delay of the transition in the boundary layer. An optimal control approach provides an efficient method for the calculation of the gradient of the aforementioned measure by solving only three adjoint equation systems, one for each of the aforementioned state equations.

The presentation is structured as follows: The state equation systems, objective functions, and systems of adjoint equations are presented in the next section. Details of the implementation, such as the mesh movement algorithm and the parameterization, are presented thereafter. This is followed by a summary of results on the accuracy of the gradients computed by the present adjoint method. In another section, the results of optimization of the airfoil RAE 2822 are presented. The first test aims at delaying only the transition, whereas a second test simultaneously seeks the reduction of the pressure drag and delay of the transition at constant lift and pitch moment coefficients. All shapes are constrained in a set of feasible designs that is presented in the implementation part. A summary and discussion section contains comments on the current achievements and on future works. The coupling of the adjoint equation systems and gradient expression are given in Appendix A.

\section{Formulation of Design Optimization}

\section{A. Inviscid Flow}

The program $\mathrm{EDGE}^{29}$ solves a node-centered and edge-based finite volume approximation of the system of Euler equations, expressed here with dimensional flow quantities. The discretization is based on a dual grid (Fig. 1). For an introduction to this type of discretization, we refer to Barth. ${ }^{30}$ In steady state, the vectors of conservative variables are solved by explicit time integration of the system

$$
V_{i} \frac{\mathrm{d} \boldsymbol{w}_{i}}{\mathrm{~d} t}+\boldsymbol{R}_{i}=0, \quad \forall i \in \mathcal{V}(\bar{\Omega})
$$

Fig. 1 Inviscid flow computation and integrated normal vector associated with edge $i j$ : - - - , dual grid.

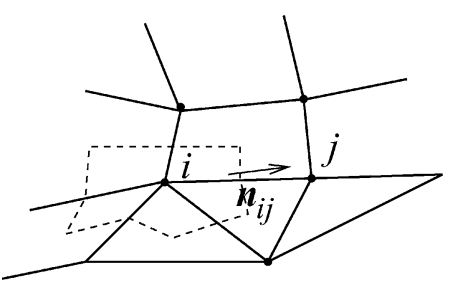


until the residuals vanish within some tolerance, where

$$
\begin{gathered}
\boldsymbol{R}_{i}=\sum_{j \in \mathcal{N}_{i}}\left(\boldsymbol{n}_{i j} \cdot \boldsymbol{f}_{i j}+\boldsymbol{d}_{i j}\right), \quad \forall i \in \mathcal{V}(\Omega) \\
\boldsymbol{R}_{i}=\sum_{j \in \mathcal{N}_{i}}\left(\boldsymbol{n}_{i j} \cdot \boldsymbol{f}_{i j}+\boldsymbol{d}_{i j}\right)+\boldsymbol{n}_{i} \cdot \boldsymbol{f}_{i}^{\mathrm{BC}}, \quad \forall i \in \mathcal{V}(\partial \Omega)
\end{gathered}
$$

The fluxes on a control surface associated with $\boldsymbol{n}_{i j}$ are approximated by $f_{i j}$ chosen here to give a central scheme because $\left(f_{i}+f_{j}\right) / 2$, where $\boldsymbol{f}_{i}=\boldsymbol{f}\left(\boldsymbol{w}_{i}\right)$ and

$$
\boldsymbol{f}_{i}=\left[\rho_{i} \boldsymbol{u}_{i}, \rho_{i} \boldsymbol{u}_{i} \otimes \boldsymbol{u}_{i}+\boldsymbol{I} p_{i}, \boldsymbol{u}_{i}\left(E_{i}+p_{i}\right)\right]^{T}
$$

Convergence to steady state is accelerated by local time stepping and the use of a multigrid. Expression (3) is calculated assuming an ideal fluid and the law of perfect gas, which yields

$$
p_{i}=(\gamma-1)\left[E_{i}-\frac{1}{2}\left(\boldsymbol{m}_{i}^{2} / \rho_{i}\right)\right]
$$

An impermeability boundary condition, $\boldsymbol{u}_{i} \cdot \boldsymbol{n}_{i}=0$, is applied on the wall boundary, yielding the boundary fluxes

$$
\boldsymbol{f}_{i}^{\mathrm{BC}}=\left[\mathbf{0}, \boldsymbol{I} p_{i}, \mathbf{0}\right]^{T}
$$

On a far-field boundary, the fluxes (3) are computed using the characteristic primitive variables based on either the far-field data for incoming characteristics, or on the flow data at the preceding time step for outgoing characteristics,

$$
\begin{aligned}
\boldsymbol{v}_{i}^{c}\left(\hat{\boldsymbol{n}}_{i}\right) & =\boldsymbol{L}\left(\hat{\boldsymbol{n}}_{i}, \boldsymbol{v}_{\infty}\right) \boldsymbol{H}\left(\lambda_{i}\right) \boldsymbol{L}^{-1}\left(\hat{\boldsymbol{n}}_{i}, \boldsymbol{v}_{\infty}\right) \boldsymbol{v}_{i}+\boldsymbol{L}\left(\hat{\boldsymbol{n}}_{i}, \boldsymbol{v}_{\infty}\right)\left(\boldsymbol{I}-\boldsymbol{H}\left(\lambda_{i}\right)\right) \\
\times & \boldsymbol{L}^{-1}\left(\hat{\boldsymbol{n}}_{i}, \boldsymbol{v}_{\infty}\right) \boldsymbol{v}_{\infty}
\end{aligned}
$$

where $\boldsymbol{L}\left(\hat{\boldsymbol{n}}_{i}, \boldsymbol{v}_{\infty}\right)$ diagonalizes the Jacobian matrix of the flux in primitive variables along the outward-directed unit normal $\hat{\boldsymbol{n}}_{i}, \boldsymbol{H}\left(\lambda_{i}\right)$ is a diagonal matrix whose diagonal is 0 for negative eigenvalues and 1 for positive ones, and $\boldsymbol{I}$ is the identity matrix. The boundary flux takes the form

$$
f_{i}^{\mathrm{BC}}=\boldsymbol{f}\left(\boldsymbol{v}_{i}^{c}\left(\hat{\boldsymbol{n}}_{i}\right)\right)
$$

An artificial dissipation flux $\boldsymbol{d}_{i j}$, a blend of second- and fourth-order differences of the Jameson type, is used.

\section{B. Viscous Flow}

The flowfield considered here is the boundary layer on a swept wing with infinite span, which is obtained by solving the mass, momentum, and energy conservation equations for a viscous compressible fluid. The equations are written in an orthogonal curvilinear coordinate system where $x^{1}, x^{2}$, and $x^{3}$ are the streamwise, spanwise, and wall-normal directions, respectively. A length element, assuming an infinite swept wing, is defined by $\mathrm{d} s^{2}=\left(h_{1} \mathrm{~d} x^{1}\right)^{2}+\left(\mathrm{d} x^{2}\right)^{2}+\left(\mathrm{d} x^{3}\right)^{2}$, where

$$
\left(h_{1}\right)^{2}=\sum_{j=1}^{3}\left(\frac{\partial X^{j}}{\partial x^{1}}\right)^{2}
$$

where $X^{j}$ are the Cartesian coordinates and $x^{i}$ the curvilinear coordinates. The total flowfield $Q_{\text {tot }}$ is decomposed into a mean $\bar{Q}$ and a perturbation part $\tilde{Q}$ as

$$
Q_{\mathrm{tot}}\left(x^{1}, x^{2}, x^{3}, t\right)=\bar{Q}\left(x^{1}, x^{3}\right)+\tilde{Q}\left(x^{1}, x^{2}, x^{3}, t\right)
$$

where $\bar{Q}$ is one of the mean variables $[\bar{U}, \bar{V}, \bar{W}, \bar{P}, \bar{T}, \bar{\rho}] \underset{\tilde{P}}{\text { and }} \tilde{Q}$ is the corresponding disturbance variable among $[\tilde{U}, \tilde{V}, \tilde{W}, \tilde{P}, \tilde{T}, \tilde{\rho}]$. The equations are derived for a quasi-three-dimensional mean flow with zero variation in the spanwise direction. The evolution of convectively unstable disturbances is analyzed in the framework of the nonlocal stability theory. All flow and material quantities are made dimensionless with the corresponding reference flow quantities at a fixed streamwise position $x_{0}$, except the pressure, which is made dimensionless with twice the corresponding dynamic pressure. The reference length scale is taken as $l_{0}=\left(v_{0} x_{0} / u_{0}\right)^{1 / 2}$, and the Reynolds number and Mach number are defined as $R e=l_{0} u_{0} / \nu_{0}$ and $M=u_{0} /\left(\mathcal{R} \gamma T_{0}\right)^{1 / 2}$, respectively. Here, $l_{0}, R e$, and $M$ are defined using dimensional quantities.

\section{Mean-Flow Equations}

The dimensionless boundary-layer equations modeling the steady viscous compressible mean flow on a swept wing with infinite span written in primitive variable form are given as

$$
\begin{aligned}
& \frac{1}{h_{1}} \frac{\partial(\bar{\rho} \bar{U})}{\partial x^{1}}+\frac{\partial(\bar{\rho} \bar{W})}{\partial x^{3}}=0 \\
& \frac{\rho \bar{U}}{h_{1}} \frac{\partial \bar{U}}{\partial x^{1}}+\bar{\rho} \bar{W} \frac{\partial \bar{U}}{\partial x^{3}}=-\frac{1}{h_{1}} \frac{\mathrm{d} \bar{P}_{e}}{\mathrm{~d} x^{1}}+\frac{1}{\operatorname{Re}} \frac{\partial}{\partial x^{3}}\left(\bar{\mu} \frac{\partial \bar{U}}{\partial x^{3}}\right) \\
& \frac{\bar{\rho} \bar{U}}{h_{1}} \frac{\partial \bar{U}}{\partial x^{1}}+\bar{\rho} \bar{W} \frac{\partial \bar{V}}{\partial x^{3}}=\frac{1}{\operatorname{Re}} \frac{\partial}{\partial x^{3}}\left(\bar{\mu} \frac{\partial \bar{V}}{\partial x^{3}}\right) \\
& \bar{c}_{p} \frac{\bar{\rho} \bar{U}}{h_{1}} \frac{\partial \bar{T}}{\partial x^{1}}+\bar{c}_{p} \bar{\rho} \bar{W} \frac{\partial \bar{T}}{\partial x^{3}}=\frac{1}{\operatorname{Re} \operatorname{Pr}} \frac{\partial}{\partial x^{3}}\left(\bar{\kappa} \frac{\partial \bar{T}}{\partial x^{3}}\right) \\
& +(\gamma-1)\left\{\frac{\bar{U} M^{2}}{h_{1}} \frac{\mathrm{d} \bar{P}_{e}}{\mathrm{~d} x^{1}}+\frac{\bar{\mu} M^{2}}{\operatorname{Re}}\left[\left(\frac{\partial \bar{U}}{\partial x^{3}}\right)^{2}+\left(\frac{\partial \bar{V}}{\partial x^{3}}\right)^{2}\right]\right\}
\end{aligned}
$$

Under the boundary-layer assumptions, the pressure is constant in the direction normal to the boundary layer, that is, $\bar{P}=\bar{P}_{e}\left(x^{1}\right)$, where subscript $e$ denotes values at the boundary-layer edge. The equation of state can then be expressed as $\gamma M^{2} \bar{P}_{e}=\bar{\rho} \bar{T}$, and the streamwise derivative of the pressure is given as

$$
\frac{\mathrm{d} \bar{P}_{e}}{\mathrm{~d} x^{1}}=-\bar{\rho}_{e} \bar{U}_{e} \frac{\mathrm{d} \bar{U}_{e}}{\mathrm{~d} x^{1}}
$$

Given a pressure distribution, given by the pressure coefficient

$$
C_{p}=\left(p_{e}-p_{\infty}\right) / \frac{1}{2} \rho_{\infty} \boldsymbol{u}_{\infty}^{2}
$$

the values at the boundary-layer edge are given as

$$
\begin{gathered}
\bar{P}_{e}=\left(p_{e} / p_{\infty}\right)\left(1 / \gamma M^{2}\right), \quad \bar{T}_{e} / \bar{T}_{\infty}=\left(\bar{P}_{e} / \bar{P}_{\infty}\right)^{(\gamma-1) / \gamma} \\
\bar{\rho}_{e} / \bar{\rho}_{\infty}=\left(\bar{P}_{e} / \bar{P}_{\infty}\right)^{1 / \gamma} \\
\bar{U}_{e}=\sqrt{ }\left(\bar{Q}_{e}^{2}-\bar{V}_{e}^{2}\right), \text { and } \bar{V}_{e}=\sin \psi, \text { where } \\
\frac{\bar{P}_{e}}{\bar{P}_{\infty}}=\frac{p_{e}}{p_{\infty}}=1+\frac{1}{2} C_{p} \gamma M^{2}, \quad \bar{Q}_{e}^{2}=1+\frac{1-\bar{T}_{e} \bar{c}_{p \infty}}{(\gamma-1) \frac{1}{2} M^{2}}
\end{gathered}
$$

Here, we have used the assumptions that for an inviscid, steady, and adiabatic flow, the total enthalpy is constant along a streamline and that the isentropic relations are used to obtain the relation between total and static quantities. A domain $\Omega_{B}$ is defined for Eqs. (8-11) such that $x^{1} \in\left[X_{S}, X_{1}\right], x^{2} \in\left[Z_{0}, Z_{1}\right]$, and $x^{3} \in[0, \infty)$. The no-slip condition is used for the velocity components, and the adiabatic wall condition is used for the temperature. In the freestream, the streamwise and spanwise velocity components and the temperature take the corresponding values at the boundary-layer edge. This can be written as

$$
\begin{gathered}
{\left[\bar{U}, \bar{V}, \bar{W}, \frac{\partial \bar{T}}{\partial x^{3}}\right]\left(x^{1}, 0\right)=[0,0,0,0], \quad \forall x^{1} \in\left[X_{S}, X_{1}\right]} \\
\lim _{x^{3} \rightarrow+\infty}[\bar{U}, \bar{V}, \bar{T}]\left(x^{1}, x^{3}\right)=\left[\bar{U}_{e}, \bar{V}_{e}, \bar{T}_{e}\right]\left(x^{1}\right), \quad \forall x^{1} \in\left[X_{S}, X_{1}\right]
\end{gathered}
$$

Equations (8-11) are integrated in the downstream direction normal to the leading edge with an initial condition given by the solution 
at the stagnation line. In the following sections, we denote the solution of the boundary-layer state $\bar{Q}=(\bar{U}, \bar{V}, \bar{W}, \bar{T})$ to simplify the presentation.

\section{Disturbance Equations}

The evolution of convectively unstable disturbances is analyzed in the framework of the nonlocal stability theory. The disturbances analyzed here are assumed to be time- and spanwise periodic waves as

$$
\tilde{\boldsymbol{Q}}\left(x^{1}, x^{2}, x^{3}, t\right)=\hat{\boldsymbol{Q}}\left(x^{1}, x^{3}\right) \Theta\left(x^{1}, x^{2}, t\right)
$$

where

$$
\Theta\left(x^{1}, x^{2}, t\right)=\exp i\left(\int_{X_{0}}^{x^{1}} \alpha\left(x^{\prime}\right) \mathrm{d} x^{\prime}+\beta x^{2}-\omega t\right)
$$

Here, $\alpha$ is the complex streamwise wave number, $\beta$ the real spanwise wave number, and $\omega$ the real disturbance angular frequency. Disturbances are superimposed on the mean flow at a streamwise position denoted $X_{0}$. We assume a scale separation $R e^{-1}$ between the weak variation in the $x^{1}$ direction and the strong variation in the $x^{3}$ direction. Furthermore, it is assumed that $\partial / \partial x^{1} \sim \mathcal{O}\left(R e^{-1}\right)$ and $W \sim \mathcal{O}\left(R e^{-1}\right)$. When the ansatz (12) and the preceding assumptions are introduced in the linearized governing equations and the terms are kept up to order $\mathcal{O}\left(R e^{-1}\right)$, a set of nearly parabolic partial differential equations, is derived. ${ }^{31-34}$ The system of equations, called parabolized stability equations (PSE), are lengthy and are, therefore, written here as

$$
\mathcal{A} \hat{\boldsymbol{Q}}+\mathcal{B} \frac{\partial \hat{\boldsymbol{Q}}}{\partial x^{3}}+\mathcal{C} \frac{\partial^{2} \hat{\boldsymbol{Q}}}{\left(\partial x^{3}\right)^{2}}+\mathcal{D} \frac{1}{h_{1}} \frac{\partial \hat{\boldsymbol{Q}}}{\partial x^{1}}=\mathbf{0}
$$

where $\bar{Q}=[\hat{\rho}, \hat{U}, \hat{V}, \hat{W}, \hat{T}]^{T}$. The coefficients of the $5 \times 5$ matrices $\mathcal{A}, \mathcal{B}, \mathcal{C}$, and $\mathcal{D}$ are found in Ref. 35. A domain $\Omega_{P}$ for Eq. (13) is defined such that $x^{1} \in\left[X_{0}, X_{1}\right], x^{2} \in\left[Z_{0}, Z_{1}\right]$, and $x^{3} \in[0, \infty)$. The boundary conditions corresponding to Eq. (13) are given as

$$
\begin{gathered}
{[\hat{U}, \hat{V}, \hat{W}, \hat{T}]\left(x^{1}, 0\right)=[0,0,0,0], \quad \forall x^{1} \in\left[X_{0}, X_{1}\right]} \\
\lim _{x^{3} \rightarrow+\infty}[\hat{U}, \hat{V}, \hat{W}, \hat{T}]\left(x^{1}, x^{3}\right)=[0,0,0,0], \quad \forall x^{1} \in\left[X_{0}, X_{1}\right]
\end{gathered}
$$

To remove the ambiguity of having $x^{1}$ dependence of both the amplitude and wave function in the ansatz, and to maintain a slow streamwise variation of the amplitude function $\hat{Q}$, a so-called auxiliary condition is introduced:

$$
\int_{0}^{+\infty} \hat{\boldsymbol{Q}}^{H} \frac{\partial \hat{\boldsymbol{Q}}}{\partial x^{1}} \mathrm{~d} x^{3}=0
$$

Equation (13) is integrated in the downstream direction normal to the leading edge with an initial condition given by local stability theory. At each $x^{1}$ position the streamwise wave number $\alpha$ is iterated such that the condition given by Eq. (14) is satisfied. After a converged streamwise wave number has been obtained, the growth rate based on the disturbance kinetic energy is calculated from the relation

$$
\sigma=-\alpha_{i}+\frac{\partial}{\partial x^{1}}(\ln \sqrt{\hat{E}})
$$

where

$$
\hat{E}=\int_{0}^{+\infty} \bar{\rho}\left(|\hat{U}|^{2}+|\hat{V}|^{2}+|\hat{W}|^{2}\right) \mathrm{d} x^{3}
$$

The growth rate can then be used to predict the transition location using the so-called $e^{N}$ method. ${ }^{4-6}$ The $N$-factor based on the disturbance kinetic energy of a single disturbance is given as

$$
N_{E}=\int_{X_{n 1}}^{X} \sigma \mathrm{d} x^{1}
$$

A complete description of Eq. (13) is found in Ref. 35, and the numerical schemes used here are given in Ref. 36.

\section{Problem of Optimal NLF Design}

As discussed in the Introduction, our approach of aerodynamic design optimization intends to reduce the viscous drag by delaying the transition in the boundary layer. The objective function must, therefore, include a measure related to the transition process. The complete aerodynamic design, however, must minimize both the viscous and pressure drag and include constraints on the lift and pitch moment coefficients as well as on the feasible geometries.

\section{Measure Related to Transition}

One choice is to measure the kinetic energy of a certain disturbance at a downstream position, for example, $X_{f}$. This can be written as

$$
E_{f}=\left.\frac{1}{2} \int_{z_{0}}^{z_{1}} \int_{0}^{+\infty} \tilde{\boldsymbol{Q}}^{H} M_{Q} \tilde{\boldsymbol{Q}} h_{1} \mathrm{~d} x^{2} \mathrm{~d} x^{3}\right|_{x^{1}=X_{f}}
$$

which means that the disturbance kinetic energy is calculated from the disturbance velocity components. If the position $X_{f}$ is chosen as the upper branch of the neutral curve, then the measure can be related to the maximum value of the $N$ factor of a given disturbance as

$$
N_{\max }=\ln \sqrt{E_{f} / E_{0}}
$$

If, in addition, the value of the $N$ factor of the measured disturbance is the one that first reaches the transition $N$ factor, then the position can be related to the onset of laminar-turbulent transition. It is, however, not clear, a priori, that minimizing such a measure will damp the chosen or other disturbances in the whole unstable region, especially if different types of disturbances are present, such as Tollmien-Schlichting (TS) and crossflow waves. For Blasius flow, it has been shown that an objective function based on a single TS wave is sufficient to damp successfully the amplification of other TS waves; see Pralits et al. ${ }^{28}$ and Airiau et al. ${ }^{37}$ On a wing, however, it is common that both TS and crossflow waves are present simultaneously.

An alternative is, therefore, to measure the kinetic energy as the streamwise integral over a defined domain. When such an approach is used, several different disturbances can be accounted for, with respective maximum growth rates at different positions. This has shown to be successful in an optimal control problem using mean flow suction as control variable; see Pralits and Hanifi. ${ }^{27}$ The size of $K$ disturbances superimposed on the mean flow at an upstream position $X_{0}$ is measured by their total kinetic energy as

$$
E_{K}=\frac{1}{2} \sum_{k=1}^{K} \int_{X_{m s}}^{X_{m e}} \int_{z_{0}}^{z_{1}} \int_{0}^{+\infty} \tilde{\boldsymbol{Q}}_{k}^{H} M_{Q} \tilde{\boldsymbol{Q}}_{k} h_{1} \mathrm{~d} x^{1} \mathrm{~d} x^{2} \mathrm{~d} x^{3}
$$

Here $X_{m s}$ and $X_{m e}$ are the first and last streamwise positions between which the disturbance kinetic energy is integrated, and they add the possibility to evaluate $E_{K}$ in a streamwise domain within $\left[X_{0}, X_{1}\right]$.

\section{Objective Functions}

In a first approach, the objective function $J$ is the total disturbance kinetic energy of a single disturbance (17), here using the disturbance that gives the largest value of $E_{1}$. The objective function depends explicitly on $\tilde{\boldsymbol{Q}}$ and on the shape of the airfoil $\Gamma$ :

$$
J=E_{1}(\equiv J(\tilde{Q}, \Gamma))
$$

Constraints are further imposed on the feasible designs to produce smooth shapes and to enforce geometric features. These are constant cross-sectional area, fixed trailing edge, and a fixed region of the airfoil around the leading edge. The last is applied to a region between 0 of the chord length and $X_{m s}$ (given in Table 1) and is meant to eliminate variations in the location of the stagnation point, which, in the current state of development of our codes, could not be accounted for in the sensitivity of the propagation of the disturbances.

A second objective function $J_{C}$ is considered to delay simultaneously the transition; reduce the pressure drag; and, in addition, 
Table 1 Optimization tests description

\begin{tabular}{lccccc}
\hline \hline Case & $\begin{array}{c}\text { Objective } \\
\text { function }\end{array}$ & $M_{\infty}$ & $\begin{array}{c}R e_{\infty}, \\
\times 10^{-6}\end{array}$ & $\begin{array}{c}{[f, \beta]} \\
{[\mathrm{kHz}, 1 / \mathrm{m}]}\end{array}$ & $\begin{array}{c}{\left[X_{m s}, X_{m e}\right]} \\
\times 10^{2}\end{array}$ \\
\hline T11 & $J\left(=E_{1}\right)$ & 0.734 & 17 & {$[15.5,0]$} & {$[4.3,45]$} \\
T12 & $J\left(=E_{1}\right)$ & 0.734 & 6.5 & {$[11,500]$} & {$[4.3,45]$} \\
T31 & $J_{C}$ & 0.734 & 17 & {$[15.5,0]$} & {$[4.3,45]$} \\
T32 & $J_{C}$ & 0.734 & 6.5 & {$[11,500]$} & {$[4.3,45]$} \\
\hline \hline
\end{tabular}

penalize changes in the coefficients of lift and pitch moment. The geometrical constraints are identical to the first type of optimization described earlier. This second objective function is defined as

$$
J_{C}=\lambda_{U} E_{1}+\lambda_{D} C_{D}+\frac{1}{2} \lambda_{L}\left(C_{L}-C_{L}^{0}\right)^{2}+\frac{1}{2} \lambda_{M}\left(C_{M}-C_{M}^{0}\right)^{2}
$$

where the drag, lift, and pitch moment coefficients are calculated only from the inviscid flow as

$$
\begin{gathered}
C_{D}=\sum_{i \in \mathcal{V}\left(\partial \Omega_{w}\right)} \frac{p_{i} \boldsymbol{n}_{i} \cdot \boldsymbol{d}_{D}}{\frac{1}{2} \rho_{\infty} \boldsymbol{u}_{\infty}^{2} S_{\mathrm{ref}}}, \quad C_{L}=\sum_{i \in \mathcal{V}\left(\partial \Omega_{w}\right)} \frac{p_{i} \boldsymbol{n}_{i} \cdot \boldsymbol{d}_{L}}{\frac{1}{2} \rho_{\infty} \boldsymbol{u}_{\infty}^{2} S_{\mathrm{ref}}} \\
C_{M}=\sum_{i \in \mathcal{V}\left(\partial \Omega_{w}\right)} \frac{p_{i} \boldsymbol{d}_{M} \cdot\left(\boldsymbol{x}_{i}-\boldsymbol{O}_{\mathrm{ref}}\right) \times \boldsymbol{n}_{i}}{\frac{1}{2} \rho_{\infty} \boldsymbol{u}_{\infty}^{2} S_{\mathrm{ref}} L_{\mathrm{ref}}}
\end{gathered}
$$

where $\boldsymbol{d}_{D}$ is a unit vector in the direction of the far-field velocity, $\boldsymbol{d}_{D}=-\boldsymbol{v}_{\infty} /\left|\boldsymbol{v}_{\infty}^{2}\right| ; \boldsymbol{d}_{L}$ is a unit vector orthogonal to $\boldsymbol{d}_{D} ;$ and $\boldsymbol{d}_{M}$ is a unit vector orthogonal to $\boldsymbol{d}_{D}$ and $\boldsymbol{d}_{L}$. Note that this second objective function depends explicitly on the modeled disturbance $\tilde{Q}$, on the shape $\Gamma$, and on the solution of the inviscid flow $\boldsymbol{w}$.

\section{Gradient Calculation}

The minimization of objective function (18) by gradient-based optimization requires the gradient, denoted $\nabla J$, with respect to the variables of design (for a given parameterization of $\Gamma$ ). It is shown in Appendix A that the adjoint approach used here enables an efficient computation of $\nabla J$. The coupling between the solutions of the three adjoint equation systems is described in the next section. Additional details of the gradient computation are discussed in the section "Implementation."

The gradient of objective function (19) is a linear combination of the gradients of $E_{1}(\equiv J), C_{D}, C_{L}$, and $C_{M}$. We refer to Amoignon ${ }^{38}$ for the calculation of the gradients of the drag, lift, and pitch moment coefficients using the discrete adjoint of the Euler equations.

The contribution from the boundary layer and stability analysis to the gradient of the objective function [the three first terms in Eq. (A31)] with respect to the surface nodes is given hereafter. A variation of the objective function with respect to the scale factors of the curvilinear coordinates is written

$$
\begin{aligned}
\delta J= & \left\langle\frac{1}{2} \tilde{\boldsymbol{Q}}_{1}^{H} M_{Q} \tilde{\boldsymbol{Q}}_{1}, \delta h_{1}\right\rangle+\left\langle\left(\frac{\partial \hat{\boldsymbol{Q}}^{*}}{\partial x^{3}}\right)^{H} \mathcal{A}_{1} \hat{\boldsymbol{Q}}\right. \\
& \left.+\hat{\boldsymbol{Q}}^{* H}\left(\mathcal{A}_{2} \frac{\partial \hat{\boldsymbol{Q}}}{\partial x^{3}}+\mathcal{A}_{3} \hat{\boldsymbol{Q}}+\mathcal{A}_{4} \frac{1}{h_{1}} \frac{\partial \hat{\boldsymbol{Q}}}{\partial x^{1}}\right), \delta h_{1}\right\rangle \\
& +\left\langle\bar{W}^{*} \frac{1}{h_{1}} \frac{\partial(\bar{\rho} \bar{U})}{\partial x^{1}}+\bar{V}^{*} \frac{\bar{\rho} \bar{U}}{h_{1}} \frac{\partial \bar{V}}{\partial x^{1}}+\bar{U}^{*}\left(\frac{\bar{\rho} \bar{U}}{h_{1}} \frac{\partial \bar{U}}{\partial x^{1}}+\frac{1}{h_{1}} \frac{\mathrm{d} P_{e}}{\mathrm{~d} x^{1}}\right)\right. \\
& \left.+\bar{T}^{*}\left(c_{p} \frac{\bar{\rho} \bar{U}}{h_{1}} \frac{\partial \bar{T}}{\partial x^{1}}-(\gamma-1) \frac{\bar{U} M^{2}}{h_{1}} \frac{\mathrm{d} P_{e}}{\mathrm{~d} x^{1}}\right), \delta h_{1}\right\rangle
\end{aligned}
$$

where

$$
\langle\cdot, \cdot\rangle=\int_{X_{0}}^{X_{1}} \int_{Z_{0}}^{Z_{1}} \int_{0}^{+\infty} d x^{1} \mathrm{~d} x^{2} \mathrm{~d} x^{3}
$$

The nonzero elements of the $5 \times 5$ matrices $\mathcal{A}_{1}, \mathcal{A}_{2}, \mathcal{A}_{3}$, and $\mathcal{A}_{4}$ are given in Appendix B. For an infinite swept wing, the scale factor $h_{1}$ can be written

$$
h_{1}=1+x^{3}\left\{\left|y_{w}^{\prime \prime}\right| /\left[1+\left(y_{w}^{\prime}\right)^{2}\right]^{\frac{3}{2}}\right\}
$$

Here, $y_{w}=y_{w}\left(x_{w}\right)$, prime denotes derivative, and $x_{w}$ and $y_{w}$ are the streamwise and wall-normal coordinates of the surface. A variation of $h_{1}=h_{1}\left[y_{w}\left(x_{w}\right)\right]$ gives

$$
\delta h_{1}=\frac{\partial h_{1}}{\partial y_{w}} \delta y_{w}+\frac{\partial h_{1}}{\partial y_{w}} \frac{\partial y_{w}}{\partial x_{w}} \delta x_{w}
$$

which is then substituted into expression (21).

The variation of the objective function corresponding to a variation of the solution of the Euler equations alone [last term in Eq. (A31)] due to a variation $\delta \boldsymbol{X}$ of the nodal coordinates in the entire Euler grid is given here in terms of the variation in the normal vectors associated with the control volumes,

$$
\delta J=-\sum_{i j \in \mathcal{E}(\bar{\Omega})}\left(\boldsymbol{w}_{i}^{*}-\boldsymbol{w}_{j}^{*}\right)^{T} \boldsymbol{f}_{i j} \cdot \delta \boldsymbol{n}_{i j}-\sum_{i \in \mathcal{V}\left(\partial \Omega_{h}\right)} \boldsymbol{w}_{i}^{* T} \boldsymbol{f}_{i}^{\mathrm{BC}} \cdot \delta \boldsymbol{n}_{i}
$$

Expression (22) is used to obtain the variation of the objective function with respect to a variation in the nodes coordinates by expressing $\delta \boldsymbol{n}$ in terms of $\delta \boldsymbol{X}$; see Amoignon. ${ }^{38}$

\section{E. Adjoint of Viscous Flow Equations}

\section{Adjoint of PSE}

The adjoint of the PSE (13) and (14) are derived using a continuous approach. The derivation in Ref. 35 yields

$$
\begin{array}{rlr}
\tilde{\mathcal{A}} \hat{\boldsymbol{Q}}^{*}+\tilde{\mathcal{B}} \frac{\partial \hat{\boldsymbol{Q}}^{*}}{\partial x^{3}}+\tilde{\mathcal{C}} \frac{\partial^{2} \hat{\boldsymbol{Q}}^{*}}{\left(\partial x^{3}\right)^{2}}+\tilde{\mathcal{D}} \frac{1}{h_{1}} \frac{\partial \hat{\boldsymbol{Q}}^{*}}{\partial x^{1}}=\boldsymbol{S}_{P}^{*} & \\
\frac{\partial}{\partial x^{1}} \int_{0}^{+\infty} \hat{\boldsymbol{Q}}^{* H}\left(\frac{\partial \mathcal{A}}{\partial \alpha}+\frac{\partial \mathcal{B}}{\partial \alpha}\right) \hat{\boldsymbol{Q}} h_{1} \mathrm{~d} x^{3} & \\
=0, & \forall x^{1} \notin\left[X_{m s}, X_{m e}\right] \\
=-i|\Theta|^{2} \int_{0}^{+\infty} \hat{\boldsymbol{Q}}^{H} M_{Q} \hat{\boldsymbol{Q}} h_{1} \mathrm{~d} x^{3}, & \forall x^{1} \in\left[X_{m s}, X_{m e}\right]
\end{array}
$$

where

$\boldsymbol{S}_{P}^{*}= \begin{cases}-\bar{\alpha}^{*} \frac{\partial \hat{\boldsymbol{Q}}}{\partial x^{1}}-\frac{\partial\left(\alpha^{*} \hat{\boldsymbol{Q}}\right)}{\partial x^{1}}, & \forall x^{1} \notin\left[X_{m s}, X_{m e}\right] \\ -\bar{\alpha}^{*} \frac{\partial \hat{\boldsymbol{Q}}}{\partial x^{1}}-\frac{\partial\left(\alpha^{*} \hat{\boldsymbol{Q}}\right)}{\partial x^{1}}+M_{Q}^{H} \hat{\boldsymbol{Q}}|\Theta|^{2}, & \forall x^{1} \in\left[X_{m s}, X_{m e}\right]\end{cases}$

and the $5 \times 5$ matrices $\tilde{\mathcal{A}}, \tilde{\mathcal{B}}, \tilde{\mathcal{C}}$, and $\tilde{\mathcal{D}}$ are found in Ref. 35 . Here, $\hat{\boldsymbol{Q}}^{*}=\left[\hat{\rho}^{*}, \bar{U}^{*}, \hat{V}^{*}, \hat{W}^{*}, \hat{T}^{*}\right]^{T}$, and the preceding equations are subject to the following boundary conditions:

$$
\begin{gathered}
{\left[\hat{U}^{*}, \hat{V}^{*}, \hat{W}^{*}, \hat{T}^{*}\right]\left(x^{1}, 0\right)=[0,0,0,0], \quad \forall x^{1} \in\left[X_{0}, X_{1}\right]} \\
\lim _{x^{3} \rightarrow+\infty}\left[\hat{U}^{*}, \hat{V}^{*}, \hat{W}^{*}, \hat{T}^{*}\right]\left(x^{1}, x^{3}\right)=[0,0,0,0], \quad \forall x^{1} \in\left[X_{0}, X_{1}\right]
\end{gathered}
$$

The initial conditions are

$$
\begin{gathered}
\hat{\boldsymbol{Q}}^{*}\left(X_{1}, x^{3}\right)=0 \quad \forall x^{3} \in[0,+\infty) \\
\alpha^{*}\left(X_{1}\right)=0 \quad \forall x^{3} \in[0,+\infty)
\end{gathered}
$$

Equation (23) is solved by backward integration in the streamwise direction. Reusing the code developed for the PSE, we solve the adjoint PSE iteratively to satisfy the auxiliary condition (24) for $\alpha^{*}$ at each streamwise position. 
2. Adjoint of Boundary-Layer Equations

The adjoint of the boundary-layer equations (8-11) are derived using a continuous approach. The derivation in Refs. 27 and 39 yields

$$
\begin{gathered}
\bar{\rho} \frac{\partial\left(h_{1} \bar{W}^{*}\right)}{\partial x^{3}}-h_{1} \bar{\rho}\left(\frac{\partial \bar{U}}{\partial x^{3}} \bar{U}^{*}+\frac{\partial \bar{V}}{\partial x^{3}} \bar{V}^{*}+\bar{c}_{p} \frac{\partial \bar{T}}{\partial x^{3}} \bar{T}^{*}\right)=S_{W}^{*} \\
\frac{\partial\left(\bar{\rho} \bar{U} \bar{U}^{*}\right)}{\partial x^{1}}+\frac{\partial\left(h_{1} \bar{\rho} \bar{W} \bar{U}^{*}\right)}{\partial x^{3}}+(\gamma-1) M^{2} \frac{\mathrm{d} \bar{P}_{e}}{\mathrm{~d} x^{1}} \bar{T}^{*} \\
-\bar{\rho}\left(\frac{\partial \bar{U}}{\partial x^{1}} \bar{U}^{*}+\frac{\partial \bar{V}}{\partial x^{1}} \bar{V}^{*}-\frac{\partial \bar{W}^{*}}{\partial x^{1}}+\bar{c}_{p} \frac{\partial \bar{T}}{\partial x^{1}} \bar{T}^{*}\right) \\
-\frac{2(\gamma-1)}{\operatorname{Re}} M^{2} \frac{\partial}{\partial x^{3}}\left(h_{1} \bar{\mu} \frac{\partial \bar{U}}{\partial x^{3}} \bar{T}^{*}\right) \\
+\frac{1}{\operatorname{Re}} \frac{\partial}{\partial x^{3}}\left(\bar{\mu} \frac{\partial\left(h_{1} \bar{U}^{*}\right)}{\partial x^{3}}\right)=S_{U}^{*} \\
\frac{\partial\left(\bar{\rho} \bar{U} \bar{V}^{*}\right)}{\partial x^{1}}+\frac{\partial\left(h_{1} \bar{\rho} \bar{W} \bar{V}^{*}\right)}{\partial x^{3}}-\frac{2(\gamma-1)}{\operatorname{Re}} M^{2} \frac{\partial}{\partial x^{3}}\left(h_{1} \bar{\mu} \frac{\partial \bar{V}}{\partial x^{3}} \bar{T}^{*}\right) \\
+\frac{1}{\operatorname{Re}} \frac{\partial}{\partial x^{3}}\left(\bar{\mu} \frac{\partial\left(h_{1} \bar{V}^{*}\right)}{\partial x^{3}}\right)=S_{V}^{*}
\end{gathered}
$$

$$
\begin{gathered}
\bar{c}_{p} \frac{\partial\left(\bar{\rho} \bar{U} \bar{T}^{*}\right)}{\partial x^{1}}+\bar{c}_{p} \frac{\partial\left(h_{1} \bar{\rho} \bar{W} \bar{T}^{*}\right)}{\partial x^{3}}+\frac{\bar{\rho} \bar{U}}{\bar{T}}\left(\frac{\partial \bar{U}}{\partial x^{1}} \bar{U}^{*}+\frac{\partial \bar{V}}{\partial x^{1}} \bar{V}^{*}-\frac{\partial \bar{W}^{*}}{\partial x^{1}}\right) \\
+\frac{\bar{\rho} \bar{U}}{\bar{T}} \bar{c}_{p} \frac{\partial \bar{T}}{\partial x^{1}} \bar{T}^{*}+\frac{\bar{\kappa}}{\operatorname{RePr}} \frac{\partial^{2}\left(h_{1} \bar{T}^{*}\right)}{\left(\partial x^{3}\right)^{2}} \\
+\frac{(\gamma-1)}{\operatorname{Re}} M^{2} \frac{\mathrm{d} \bar{\mu}}{\mathrm{d} \bar{T}}\left[\left(\frac{\partial \bar{U}}{\partial x^{3}}\right)^{2}+\left(\frac{\partial \bar{V}}{\partial x^{3}}\right)^{2}\right] \bar{T}^{*} \\
-\frac{1}{\operatorname{Re}} \frac{\mathrm{d} \bar{\mu}}{\mathrm{d} \bar{T}}\left[\frac{\partial \bar{U}}{\partial x^{3}} \frac{\partial\left(h_{1} \bar{U}^{*}\right)}{\partial x^{3}}+\frac{\partial \bar{V}}{\partial x^{3}} \frac{\partial\left(h_{1} \bar{V}^{*}\right)}{\partial x^{3}}\right]=S_{E}^{*}
\end{gathered}
$$

where the right-hand side $S_{B}^{*}=\left[S_{W}^{*}, S_{U}^{*}, S_{V}^{*}, S_{E}^{*}\right]^{T}$ is given as

$$
\boldsymbol{S}_{B}^{*}= \begin{cases}{\left[F_{W}, F_{U}, F_{V}, F_{T}+F_{W}(\bar{W} / \bar{T})\right]^{T} h_{1},} & \forall x^{1} \in\left(X_{0}, X_{1}\right) \\ \mathbf{0}, & \forall x^{1} \in\left(X_{S}, X_{0}\right]\end{cases}
$$

The nonzero right-hand side is the coupling between the adjoint parabolized stability equations (APSE) and the adjoint boundarylayer equations (ABLE) and expresses the sensitivity of the PSE with respect the variations in $\bar{W}, \bar{U}, \bar{V}$, and $\bar{T}$, respectively. A detailed description is found in Ref. 39. The preceding equations are subject to the boundary conditions

$$
\begin{array}{rr}
{\left[\bar{U}^{*}, \bar{V}^{*}, \frac{\partial\left(h_{1} \bar{T}^{*}\right)}{\partial x^{3}}\right]\left(x^{1}, 0\right)=[0,0,0],} & \forall x^{1} \in\left[X_{0}, X_{1}\right] \\
\lim _{x^{3} \rightarrow+\infty}\left[\bar{U}^{*}, \bar{V}^{*}, \bar{W}^{*}, \bar{T}^{*}\right]\left(x^{1}, x^{3}\right)=[0,0,0,0], & \forall x^{1} \in\left[X_{0}, X_{1}\right]
\end{array}
$$

Equations (25-28) are solved by backward integration in the streamwise direction with the initial condition given at $x^{1}=X_{1}$ as

$$
\overline{\mathbf{Q}}^{*}\left(X_{1}, x^{3}\right)=\mathbf{0} \quad \forall x^{3} \in[0,+\infty)
$$

The Euler and the boundary-layer equations are coupled through the pressure distribution $P_{e}$ and the mesh given by the nodal coordinates $\boldsymbol{X}$. A variation of the geometry that affects the Euler solution will, therefore, appear as variations of the pressure distribution in the boundary-layer equations, which consequently will affect the solution of the stability equations. Pralits et al. ${ }^{28}$ considered an optimal control problem using the pressure distribution as control variables and the total disturbance kinetic energy as the objective function. From the coupled APSE and ABLE for incompressible flows, an expression was derived for the gradient of the objective function with respect to the pressure distribution. From the present APSE and
ABLE, a similar expression valid $\forall x^{1} \in\left(X_{S}, X_{1}\right)$ can be evaluated as

$$
\frac{\partial J}{\partial \bar{P}}=\int_{0}^{+\infty}\left[-\frac{\partial \bar{U}^{*}}{\partial x^{1}}+(\gamma-1) M^{2} \frac{\partial\left(\bar{T}^{*} \bar{U}\right)}{\partial x^{1}}\right] \mathrm{d} x^{3}
$$

Setting the Mach number equal to zero in expression (29), we find exactly the same expression as the one derived in Ref. 28. Note that a variation of $\boldsymbol{X}$ will also affect the nodal coordinates of the boundary-layers equations (BLE) and PSE. This can be seen in expression (A31) in Appendix A.

\section{F. Adjoint of Inviscid Flow Equations}

The adjoint of the discretized Euler equations (1) and (2) are solved following the same technique used for solving the Euler equations, namely, explicit time integration of the system

$$
V_{i} \frac{\mathrm{d} \boldsymbol{w}_{i}^{*}}{\mathrm{~d} t}+\boldsymbol{R}_{i}^{*}=\mathbf{0}, \quad \forall i \in \mathcal{V}(\bar{\Omega})
$$

until the residuals $\boldsymbol{R}_{i}^{*}$ vanish within some tolerance. The complete derivation based on discrete sensitivities can be found in Ref. 38 . The following gives expression for the adjoint residuals:

$$
\begin{aligned}
\boldsymbol{R}_{i}^{*}= & \sum_{j \in \mathcal{N}_{i}}\left[\frac{\partial\left(\boldsymbol{f}_{i} \cdot \boldsymbol{n}_{i j}\right)}{\partial \boldsymbol{w}_{i}}\right]^{T} \frac{\left(\boldsymbol{w}_{i}^{*}-\boldsymbol{w}_{j}^{*}\right)}{2}+\sum_{j \in \mathcal{N}_{i}} \boldsymbol{d}_{i j}^{*}, \quad \forall i \in \mathcal{V}(\Omega) \\
\boldsymbol{R}_{i}^{*}= & \sum_{j \in \mathcal{N}_{i}}\left[\frac{\partial\left(\boldsymbol{f}_{i} \cdot \boldsymbol{n}_{i j}\right)}{\partial \boldsymbol{w}_{i}}\right]^{T} \frac{\left(\boldsymbol{w}_{i}^{*}-\boldsymbol{w}_{j}^{*}\right)}{2}+\sum_{j \in \mathcal{N}_{i}} \boldsymbol{d}_{i j}^{*} \\
& +\left[\frac{\partial\left(\boldsymbol{f}_{i}^{\mathrm{BC}} \cdot \boldsymbol{n}_{i}\right)}{\partial \boldsymbol{w}_{i}}\right]^{T} \boldsymbol{w}_{i}^{*}, \quad \forall i \in \mathcal{V}(\partial \Omega) \\
\boldsymbol{R}_{i}^{*}= & \sum_{j \in \mathcal{N}_{i}}\left[\frac{\partial\left(\boldsymbol{f}_{i} \cdot \boldsymbol{n}_{i j}\right)}{\partial \boldsymbol{w}_{i}}\right]^{T} \frac{\left(\boldsymbol{w}_{i}^{*}-\boldsymbol{w}_{j}^{*}\right)}{2}+\sum_{j \in \mathcal{N}_{i}} \boldsymbol{d}_{i j}^{*} \\
& +\left[\frac{\partial\left(\boldsymbol{f}_{i}^{\mathrm{BC}} \cdot \boldsymbol{n}_{i}\right)}{\partial \boldsymbol{w}_{i}}\right]^{T} \boldsymbol{w}_{i}^{*}-\boldsymbol{g}_{i}^{*}, \quad \forall i \in \mathcal{V}\left(\partial \Omega^{o}\right)
\end{aligned}
$$

where $\mathcal{V}\left(\partial \Omega^{\circ}\right)$ is the set of nodes at which the pressure $P_{e}$ is measured, according to the definition of the BLE (8-11). The term $\boldsymbol{g}_{i}^{*}$ in Eq. (31) relates the solution of the ABLE, via Eq. (29), to the adjoint of the inviscid flow equations (30). This term is defined in Appendix A as the right-hand side of Eq. (A30). The flux $\boldsymbol{d}_{i j}^{*}$, adjoint of the artificial dissipation flux $\boldsymbol{d}_{i j}$, is obtained by freezing the artificial viscosities; see Amoignon. ${ }^{38}$ A similar freezing of the coefficients in the far-field boundary conditions yields the following expression for the Jacobian of the far-field flux:

$$
\frac{\partial\left(f_{i}^{\mathrm{BC}} \cdot \boldsymbol{n}_{i}\right)}{\partial \boldsymbol{w}_{i}}=\frac{\partial\left(\boldsymbol{f} \cdot \boldsymbol{n}_{i}\right)}{\partial \boldsymbol{v}_{i}} \boldsymbol{L}\left(\hat{\boldsymbol{n}}_{i}, \boldsymbol{v}_{\infty}\right) \boldsymbol{H}\left(\lambda_{i}\right) \boldsymbol{L}^{-1}\left(\hat{\boldsymbol{n}}_{i}, \boldsymbol{v}_{\infty}\right) \frac{\mathrm{d} \boldsymbol{v}_{i}}{\mathrm{~d} \boldsymbol{w}_{i}}
$$

The Jacobian of the Euler wall flux function is

$$
\frac{\partial\left(\boldsymbol{f}_{i}^{\mathrm{BC}} \cdot \boldsymbol{n}_{i}\right)}{\partial \boldsymbol{w}_{i}}=(\gamma-1)\left[\frac{1}{2}\left|\boldsymbol{u}_{i}\right|^{2},-\boldsymbol{u}_{i}, 1\right]^{T}
$$

\section{Implementation}

Objective function (18) depends on the shape, defined by the nodal coordinates $\boldsymbol{X}$, and, implicitly, on the pressure $P_{e}$ calculated from the discretized Euler equations (1) and (2). As mentioned earlier, the adjoint of the Euler equations is derived based on the discrete sensitivities. Thus, any modification of the mesh may perturb the solution of the inviscid flow, which, in turn, would perturb the solutions of the BLE, the PSE, and the value of $J$. The gradient of $J$ as a function of $\boldsymbol{X}$ is denoted $\nabla J_{X}$ in Appendix A. 
For the sake of simplicity, each node on the airfoil can only be displaced in the direction normal to the airfoil at initial design. Furthermore, to retain mesh quality, when the airfoil shape is changed, we use a mesh movement mapping to deform the mesh also inside the domain of computation for the Euler equations. In the following text, an adjoint of the mesh movement mapping yields the gradient $\nabla J_{y}$ of $J$ as a function of the normal displacements on the airfoil $y$. We use a particular parameterization of the airfoil normal displacements $\boldsymbol{y}$ that simultaneously imposes the geometric constraints and enforces smoothness. An adjoint of the mapping that defines the parameterization yields an expression for the gradient of $J$ with respect to the variables of design $\boldsymbol{a}$, denoted $\nabla J_{a}$.

\section{A. Mesh Displacements}

In the current study, an explicit affine mapping is used to propagate changes smoothly in the geometry to the entire Euler mesh. It is formulated as

$$
\boldsymbol{X}^{k}=\boldsymbol{X}^{0}+L \boldsymbol{y}^{k}
$$

Given a function $J_{X}$ of the nodal coordinates, expression (34) may be used to define a function $J_{y}(\boldsymbol{y}) \equiv J_{X}(\boldsymbol{X}(\boldsymbol{y}))$ of the normal displacements $\boldsymbol{y}$ on the airfoil. The chain rule yields the gradient of $J_{y}$ by the matrix-vector product

$$
\nabla J_{y}=L^{T} \nabla J_{X}
$$

We used a C-type mesh, a mesh that has smooth grid lines normal to the airfoil, because it makes the derivation of sparse matrices $L$ easier; see Amoignon et al. ${ }^{40}$ for details. Other mesh deformation algorithms will be necessary for meshes not having a special structure.

\section{B. Parameterization and Geometric Constraints}

In shape optimization, the combination of gradient methods and piecewise polynomial interpolations, such as B-splines, may induce oscillations in the shapes, as investigated by Frank and Shubin. ${ }^{41}$ In the current approach, smooth shapes are obtained, together with (linear) geometric constraints, by taking the vectors of normal displacements $\boldsymbol{y}$ as solution of a quadratic programming problem $(\mathrm{QP})^{38}$ of the form

$$
\boldsymbol{y}=\left\{\begin{array}{l}
\min _{v \in \mathbb{R}^{n}} \frac{1}{2} \boldsymbol{v}^{T} A_{s} \boldsymbol{v}-\boldsymbol{v}^{T} M_{s} \boldsymbol{a} \\
C^{T} \boldsymbol{v}=\boldsymbol{b}
\end{array}\right.
$$

where the columns of $C$ are the gradients of the constraints imposed on the displacements. In the case without geometric constraints $[m=0$ in Eq. (36)], the solution to Eq. (36) is solution of the discretized Poisson problem

$$
A_{s} \tilde{\boldsymbol{y}}=M_{s} \boldsymbol{a}
$$

along the surface of the airfoil. Adding constraints $[m \geq 1$ in Eq. (36)] means that the solution of Eq. (36) fulfills exactly the relations

$$
C^{T} \boldsymbol{y}=\boldsymbol{b}
$$

and, according to some norm, $\boldsymbol{y}$ is the closest to the solution of the discretized Poisson problem. It is a known result of optimization theory, ${ }^{42}$ that the solution of the QP (36) is obtained by solving the Karush-Kuhn-Tucker system

$$
\left(\begin{array}{cc}
A_{s} & -C \\
-C^{T} & 0
\end{array}\right)\left(\begin{array}{l}
\boldsymbol{y} \\
\lambda
\end{array}\right)=\left(\begin{array}{c}
M_{s} \boldsymbol{a} \\
-\boldsymbol{b}
\end{array}\right)
$$

When expression (39) is used, the design variables becomes the right-hand-side vectors $\boldsymbol{a}$ and $\boldsymbol{b}$. Given the gradient $\nabla J_{y}$ of expression (35), we can calculate the gradient with respect to the design variables $\boldsymbol{a}$ and $\boldsymbol{b}$ by first solving the adjoint problem

$$
\left(\begin{array}{cc}
A_{s}^{T} & -C \\
-C^{T} & 0
\end{array}\right)\left(\begin{array}{l}
y^{*} \\
\lambda^{*}
\end{array}\right)=\left(\begin{array}{c}
\nabla J_{y} \\
\mathbf{0}
\end{array}\right)
$$

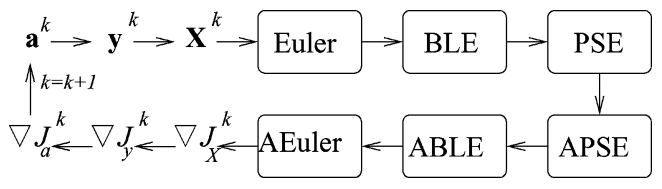

Fig. 2 Flowchart for case of minimizing disturbance kinetic energy without aerodynamic constraints.

and then evaluating

$$
\nabla J_{a}=M_{s}^{T} y^{*}, \quad \nabla J_{b}=-\lambda^{*}
$$

see Amoignon. ${ }^{38}$

\section{Optimization Algorithm}

Aerodynamic constraints (lift and pitch), when used, are incorporated in the objective function via a simple penalization technique (19). Geometrical constraints (fixed volume, fixed trailing edge, and fixed geometry around a portion of the leading edge) are handled via the preceding parameterization. Furthermore, the geometric constraints are here equalities, so that the right-hand side $\boldsymbol{b}$ in Eq. (36) is a constant vector. The only control parameter used in our applications is, therefore, the vector $\boldsymbol{a}$.

This unconstrained optimization algorithm can, therefore, be solved using a regular quasi-Newton method [Broyden-FletcherGoldfarb-Shanno (BFGS) method] with a line search algorithm.

Figure 2 shows the order of the various computations that are needed for derivative calculations in the optimal NLF design. The design $k+1$ is obtained from the optimization routine after possibly one or several objective functions and only one gradient evaluation due to the use of the Goldstein conditions in the line search algorithm; see Nocedal and Wright. ${ }^{42}$

\section{Numerical Tests}

Sufficient gradient accuracy is a critical issue in optimization. The first-order necessary optimality condition is satisfied if the gradient of the objective function or the Lagrangian vanishes. Difficulties related to low accuracy, such as to find descent directions even far from the optimal design, are quite common. There are two possible sources of inaccuracy in our calculation of the gradient. As mentioned earlier, the derivation of the adjoint of the discretized Euler equations makes use of an approximation because it does not linearize the coefficients of the second-order artificial dissipation. Moreover, the adjoint equations of the BLE and PSE are derived from the continuous state equations. Numerical tests are carried out in Ref. 40.

The accuracy of the gradient at a design point $\boldsymbol{a}$ can be analyzed by comparing the value obtained by computation of adjoint problems $\nabla J_{a}$ with an estimate by finite differences (FD),

$$
\left(\nabla J_{a}\right)_{k}^{\mathrm{FD}} \approx \frac{J_{a}\left(\boldsymbol{a}+\epsilon_{a} \boldsymbol{e}_{k}\right)-J_{a}\left(\boldsymbol{a}-\epsilon_{a} \boldsymbol{e}_{k}\right)}{2 \epsilon_{a}}
$$

where $\boldsymbol{e}_{k}$ is the vector having component $k=1$ with all other components being 0 . Several calculations of $\left(\nabla J_{a}\right)_{k}^{\mathrm{FD}}$ are commonly performed, using different values of $\epsilon_{a}$ to find the best compromise between accuracy and rounding errors, the last being inherent to the FD method. The relative error between the gradient obtained by the adjoint method $\nabla J_{a}$ and the one approximated by FD $\nabla J_{a}$ can be calculated as

$$
\operatorname{err}_{\nabla J_{a}}=\frac{\left\|\nabla J_{a}-\left(\nabla J_{a}\right)^{\mathrm{FD}}\right\|}{\left\|\left(\nabla J_{a}\right)^{\mathrm{FD}}\right\|}
$$

where \|\| denotes the norm in $\mathbb{R}^{n}$ defined by the dot product.

The tests are performed using a C-type mesh of the RAE 2822 airfoil at Mach number $M_{\infty}=0.734$. The size of the mesh is 13,352 nodes with 224 nodes on the airfoil. FD approximations of the gradient given by expression (42) are noted FD. The objective function 


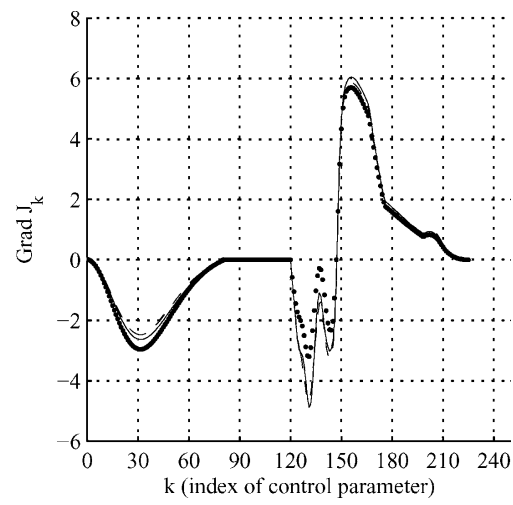

Fig. 3 Gradients of disturbance kinetic energy calculated by the methods: - FD; $\cdots$, ADJ1; and $\cdots \cdot$. , ADJ2; ADJ3 is not shown; $M_{\infty}=\mathbf{0 . 7 3 4}$.

in all tests performed here is Eq. (18). The gradient $\nabla J_{a}$ is calculated from the gradient $\nabla J_{X}$ Eq. (A31) as described in the preceding section. The gradient $\nabla J_{X}$ is given by expression (A31),

$$
\nabla J_{X}=\underbrace{\frac{\partial J_{\bar{Q}}}{\partial \boldsymbol{X}}-\left(\frac{\partial \mathcal{A}_{q}}{\partial \boldsymbol{X}}\right)^{*} \tilde{\boldsymbol{Q}}^{*}+\left(\frac{\partial \mathcal{A}_{Q}}{\partial \boldsymbol{X}}\right)^{*} \overline{\boldsymbol{Q}}^{*}}_{\text {continuous }}-\underbrace{\left(\frac{\partial \mathcal{A}_{w}}{\partial \boldsymbol{X}}\right)^{*} \boldsymbol{w}^{*}}_{\text {discrete }}
$$

As outlined earlier, the gradient is evaluated from three systems of adjoint equations that are solved in the following order:

$$
\underbrace{\text { APSE } \rightarrow \text { ABLE }}_{\text {continuous }} \rightarrow \underbrace{\text { adjoint Euler }}_{\text {discrete }}
$$

The APSE and ABLE are derived using the continuous approach, whereas the adjoint Euler is derived using the discrete approach. The influence on the accuracy of the different approaches and of the different terms in the preceding gradient expression is summarized here by comparing different ways of calculating $\nabla J_{X}$ (and, therefore, $\nabla J_{a}$ ):

1) For ADJ1, the terms denoted continuous in the preceding expression of $\nabla J_{X}$ are neglected. The error (43) is $17.2 \%$.

2) For ADJ2, $\nabla J_{X}$ is computed as in FD-ADJ1, but the righthand side $(\partial J / \partial \boldsymbol{w})$ of the adjoint Euler equations is approximated by FD, where the pressure on the shape is considered as a design variable for the system BLE-PSE. The error (43) is $4.95 \%$.

3) For ADJ3, the right-hand side of the adjoint Euler equations is computed as in FD-ADJ2, and the terms denoted continuous in the preceding expression of $\nabla J_{X}$ are calculated by FD. The error (43) is reduced by $0.1 \%$ compared to that obtained by comparing FD with ADJ2.

The gradients obtained by the methods FD, ADJ1, and ADJ2 are shown in Fig. 3. The use of FD for computing the right-hand side of the adjoint Euler equations is a discrete approach. Therefore, the comparison of the test FD-ADJ1 with the test FD-ADJ2 indicates the error due to the use of a continuous approach for the derivation of the adjoint systems ABLE and APSE. This error is here largely due to a too coarse distribution of the nodes in the streamwise direction when solving the BLE, PSE, and their adjoint systems, as discussed in Ref. 40.

The test FD-ADJ3, compared to FD-ADJ2, indicates that neglecting the terms denoted continuous in $\nabla J_{X}$ has only small effects.

In the following text, $\nabla J_{X}$ is computed as in FD-ADJ1, that is, neglecting the explicit continuous terms (terms indicated as continuous in the preceding expression of $\nabla J_{X}$ ) and computing the right-hand side of the adjoint Euler equations from the solutions of the adjoint PSE and BLE.

\section{Optimization Results}

Results are presented here for the minimization of the objective functions presented earlier. The discretization of the three state equations are based on the grid described in the preceding section. The test cases are summarized in Table 1 . The thermodynamic proper-

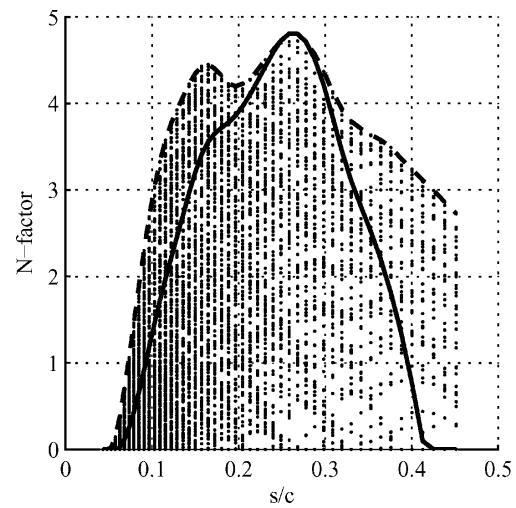

Fig. $4 \mathrm{~N}$-factor values for 165 modes (dots) for cases T12 and T32 (Table 1) at original design.

ties for the different cases correspond to two different altitudes such that the TX1 cases are given at 0-m above sea level (ASL) and the TX2 cases are given at 9600-m ASL. Note here that upstream and downstream positions of the domain where the stability equations are solved are chosen as $X_{0}=X_{m s}$ and $X_{1}=X_{m e}$.

\section{A. Analysis of Disturbance Amplification}

A stability analysis of a large number of modes with different frequencies $f$ and spanwise wave numbers $\beta$, corresponding to different wave angles, is performed before each optimization case, on the original design and for the chosen flow conditions. The corresponding $N$ factors are calculated from these results, and the optimization uses the mode that has the largest $N$-factor value with respect to all other modes. The reason of this particular choice is that it has been shown in previous studies on optimal control ${ }^{27,28}$ that a control that successfully decreases the amplification of a single disturbance also has a damping effect on other instability waves of the same type. It is common in transition prediction to compute the envelope or envelopes (EOE) of the $N$-factor curves, that is, the envelope over both frequency and spanwise wave number. Transition is then assumed to occur at the position where the EOE curve first attains an empirically determined value. This curve also serves as a measure of the efficiency of a control or design, computed by minimizing a single disturbance on a large number of disturbances.

An example of results from the analysis discussed here is shown in Fig. 4. The example concerns the initial design with a freestream Mach number $M_{\infty}=0.734$, Reynolds number $R e_{\infty}=6.5 \times 10^{6}$, and angle of attack $\alpha_{l}=2.1875 \mathrm{deg}$. A total of 165 modes have been analyzed with dimensional frequency $f=[5,20] \mathrm{kHz}$ $(\Delta f=1 \mathrm{kHz})$ and spanwise wave number $\beta=[0,2500] 1 / \mathrm{m}$ $(\Delta \beta=2501 / \mathrm{m})$. This choice of spanwise wave number corresponds to wave angles between 0 and $85 \mathrm{deg}$. The wave angle is defined as the angle between the wave number vector $\boldsymbol{k}$ and the inviscid streamline. The corresponding $N$-factor values of all modes are given by dots. The mode chosen to be used in the optimization is given by the solid line and the EOE curve by the dash line. The values of $f$ and $\beta$ given here are used for all EOE analyses made in this paper.

\section{B. Analysis of Results Using Reynolds-Averaged} Navier-Stokes Calculations

Viscous calculations using the Reynolds-averaged Navier-Stokes (RANS) equations are also carried out with EDGE $^{23}$ before and after the optimization tests. These results are used to compare the $N$ factors based on the pressure distribution obtained from the viscous calculation with those that are computed using the Euler pressure distribution. The turbulence model used in the RANS calculations is the EARSM by Wallin and Johansson ${ }^{43}$ together with the $k-\omega$ model. These calculations are also used to indicate what degree of reduction of the viscous drag can be achieved by the present approach. The C-type grid for the RANS calculations has the size 22,088 nodes with 224 nodes on the airfoil.

An attempt has been made to use the $N$-factor results of the initial and final design to determine the respective transition location. 
These results have been used as input to the RANS calculations of the initial and final design.

The transition position on the upper side of the initial design was taken as the streamwise position corresponding to the maximum value of the EOE curve of the $N$ factors computed using the pressure distribution from the Euler solution. Because the EOE curves of the final designs were lower in magnitude compared to the initial ones, the transition location of the final designs was set as the downstream position of the computational domain of the boundary layer and stability analysis. The same transition position was used on the lower side, both for the initial and final designs. Note that the transition location for the initial design is not based on experimental results. A common reference for the RAE 2822 airfoil is Ref. 44, in which the boundary layer was tripped at $3 \%$ chord in the experiments to have a well-defined turbulent portion.

\section{Minimization of $\boldsymbol{J}$, Equation (18)}

\section{Case T11}

Results of the T11 case are shown in Fig. 5. The convergence history is given in terms of the objective function and gradient

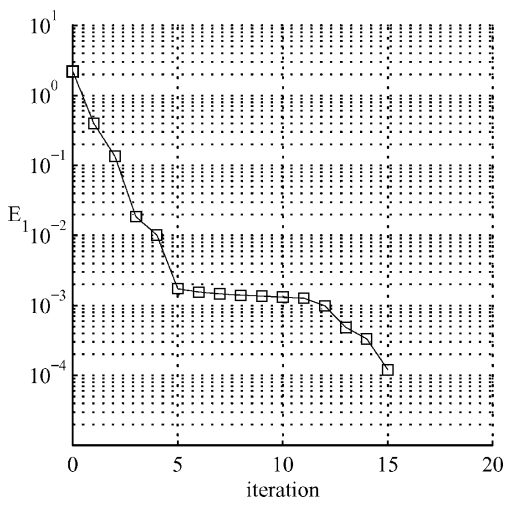

a) Objective function

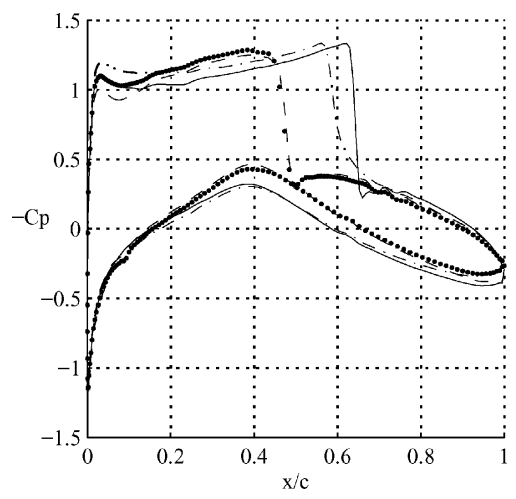

b) Pressure coefficients norm as functions of the iteration number. The optimization was stopped when the BLE solver failed to converge. This occurred as the changes in the geometry caused the shock wave to move into the computational domain of the BLE. The strong adverse pressure gradient due to the shock wave cannot be handled using the BLE because they do not model separation. This can be seen in Fig. 5, where the pressure coefficient and geometry for the initial and final design are plotted. At final design, the central upper part of the wing is thinner, measuring the thickness as the distance of a point on the airfoil to the chord. Therefore, because of the fixed region around the leading edge, the region between $4.3 \%$ of the chord length, from the leading edge, up to about $30 \%$ of the chord length, situated on the upper part, has a higher curvature at final design than at initial design. An increase of the curvature of a wall boundary is known to reduce the pressure in the fluid flow. This may be the effect that can be observed in Fig. 5, where the pressure coefficient at final design has decreased $\left(-C_{p}\right.$ is increased) in the region between $10 \%$ of the chord length, from the leading edge, up to about $30 \%$ of the chord length, in comparison to the initial design. In this way, a pressure gradient is obtained that damps the amplification of disturbances as

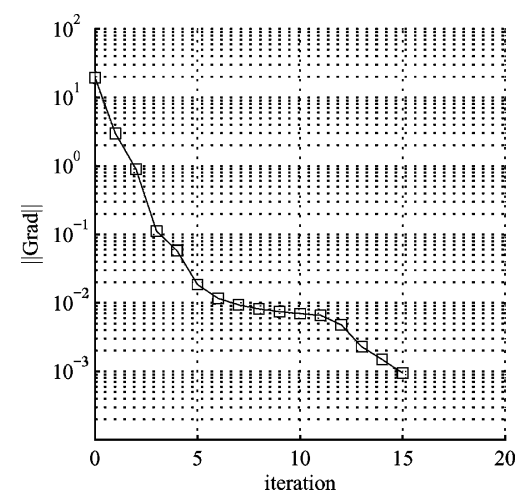

c) Norm of gradient of objective function

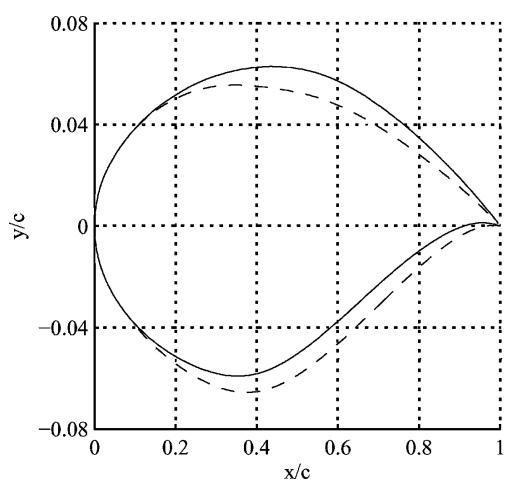

d) Airfoils

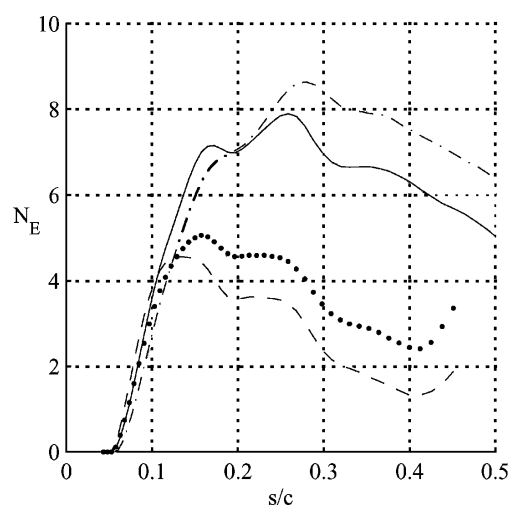

e) Envelope of envelope of $N$-factor curves

Fig. 5 T11, comparison between ——, initial and - - - , final design; pressure and EOE of $N$-factor curves involving RANS equations for -•---, initial and $\cdots \cdots$, final design. 
is explained hereafter. However, the faster decrease of the pressure may be responsible for the shock moving upstream. Note that the deformation of the lower part of the wing is only due to the constraint that imposes a constant volume. The effect on the disturbance amplification can be seen in Fig. 5, where the EOE curves have been plotted for the initial and final design. A large damping of all modes has been achieved. This can be explained by the change in pressure gradient from adverse to favorable in a large part of the region where the disturbances are amplified. Values of the drag, lift, and pitch moment coefficients for the initial and optimized designs are summarized in Table 2. The viscous drag, obtained from the RANS calculations, is reduced by six drag counts (where one drag count is $10^{-4}$ ), and, as could be expected from earlier observations on the position of the shock, the pressure drag is also decreased. However, this decrease is a byproduct of the reduction of the disturbance kinetic energy. Large changes in the lift and the pitch moment coefficients are observed, and these effects are controlled by imposing constraints in case T31.
Table 2 Comparison of aerodynamic coefficients at initial and final design for cases T11 and T31 (0 m ASL), using Euler and RANS flow analysis

\begin{tabular}{lcccr}
\hline \hline & \multicolumn{3}{c}{ RANS } & \multicolumn{1}{c}{$\begin{array}{c}\text { Euler, } \\
\text { pressure }\end{array}$} \\
\cline { 2 - 4 } Case & Total & Viscous & Pressure & \\
\hline & & ${\text { Coefficient } C_{D}}$ & & \\
Initial & $2.09 \times 10^{-2}$ & $3.35 \times 10^{-3}$ & $1.76 \times 10^{-2}$ & $1.3 \times 10^{-2}$ \\
T11 (final) & $1.53 \times 10^{-2}$ & $2.71 \times 10^{-3}$ & $1.26 \times 10^{-2}$ & $8.83 \times 10^{-3}$ \\
T31 (final) & $1.78 \times 10^{-2}$ & $2.36 \times 10^{-3}$ & $1.54 \times 10^{-2}$ & $1.04 \times 10^{-3}$ \\
& & Coefficient C $_{L}$ & & \\
Initial & $8.39 \times 10^{-1}$ & $-7.9 \times 10^{-5}$ & $8.39 \times 10^{-1}$ & $8.4 \times 10^{-1}$ \\
T11 (final) & $6.34 \times 10^{-1}$ & $-5.62 \times 10^{-5}$ & $6.34 \times 10^{-1}$ & $6.21 \times 10^{-1}$ \\
T31 (final) & $8.48 \times 10^{-1}$ & $-5.03 \times 10^{-5}$ & $8.48 \times 10^{-1}$ & $8.43 \times 10^{-1}$ \\
& & Coefficient C $_{M}$ & & \\
Initial & $3.15 \times 10^{-1}$ & - & - & $3.4 \times 10^{-1}$ \\
T11 (final) & $2.08 \times 10^{-1}$ & - & - & $2.19 \times 10^{-1}$ \\
T31 (final) & $3.18 \times 10^{-1}$ & - & - & $3.37 \times 10^{-1}$ \\
\hline \hline
\end{tabular}

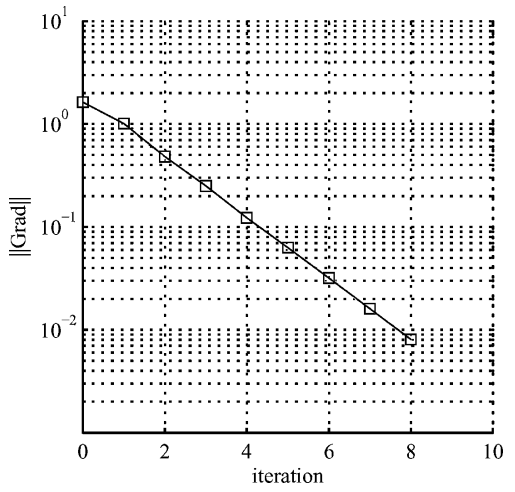

c) Norm of gradient of objective function

a) Objective function

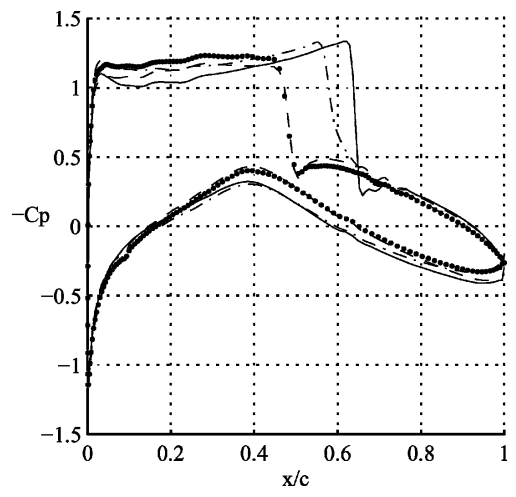

b) Pressure coefficients

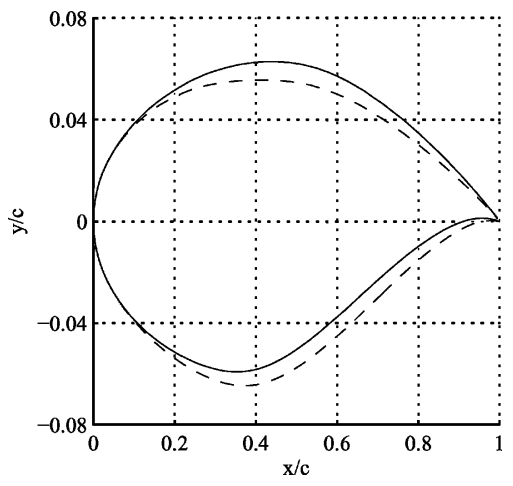

d) Airfoils

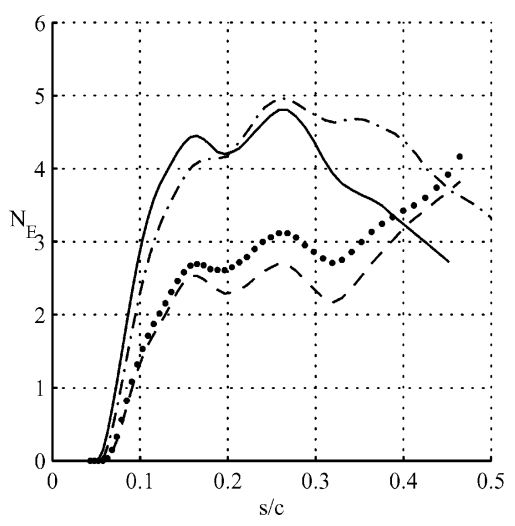

e) Envelope of envelope of $N$-factor curves

Fig. 6 T12, comparison between —_, initial and - - - , final design; pressure and EOE of $N$-factor curves involving RANS equations for -•---, initial and $\cdots \cdot$, final design. 


\section{Case T12}

Results of case T12 are given in Fig. 6. The decrease of the objective function is of one order of magnitude smaller than it is for case T11 (Fig. 5). The optimization is terminated here because no further descent direction could be found. The magnitudes of the deformations of the airfoil are smaller compared to case T11, but the trend is similar. The change of the pressure gradient may be caused by the local increase of the curvature on the upper part of the wing, between 4.3 and $30 \%$ of the chord length downstream of the leading edge. As a consequence, the shock is moved upstream. The effect on the disturbance amplification can be seen in Fig. 6, where the EOE curves have been plotted for the initial and final design. A large decrease in disturbance amplification is obtained using the optimized design, similar to the one found for the high Reynolds number case T11. Also, this is due to the change in pressure gradient from an adverse to favorable in the upstream part of the domain where the disturbances become unstable. The shock wave has moved as far upstream as in case T11.

Values of the drag, lift, and pitch-moment coefficients for the initial and optimized designs are summarized in Table 3 . The viscous drag is there reduced by 6 drag counts, and, as in case T11, the pressure drag is decreased and large changes occur in the lift and the pitch moment coefficients.

\section{Minimizing $J_{C}$, Equation (19)}

\section{Case T31}

Results of case T31 are shown in Fig. 7. The objective function is decreased in each step of the optimization, even though the component of the wave drag is increased between iteration numbers 1 and 2. The reason for the increase is that in this interval the deviation of lift and pitch-moment coefficients is decreased. A reduction has been obtained at the last iteration in both disturbance kinetic energy and wave drag, whereas the lift and pitch moment coefficients are kept within $1 \%$. The shock wave on the upper side has moved upstream and is weakened. In comparison with case T11, which has the same initial conditions, the displacement of the shock in case T31 is smaller, but the changes in case T31 preserve the pitch-moment and lift coefficients near to their values at initial design.

The effect on the disturbance amplification can be seen in Fig. 7, where the EOE curves have been plotted for the initial and final design. A damping of the disturbance amplification is obtained in a large part of the computational domain at final design. Note from Fig. 7 that the adverse pressure gradient of the initial design, in the upstream region where the disturbances become unstable, has become zero or weakly favorable in the final design. Close to the shock wave of the final design, which has now moved farther upstream,

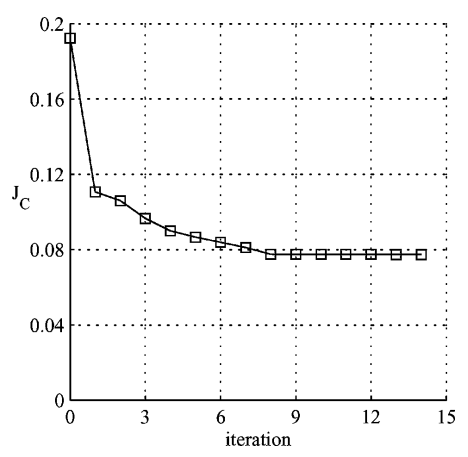

a) Objective function

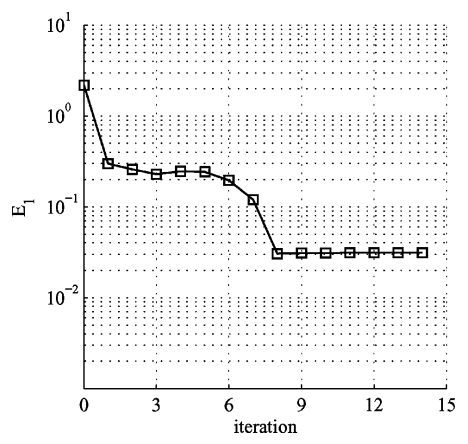

b) Disturbance kinetic energy

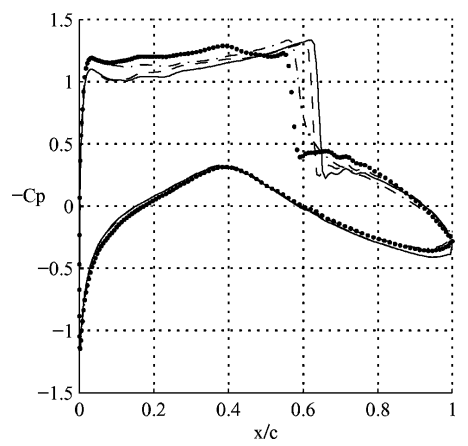

c) Pressure coefficients

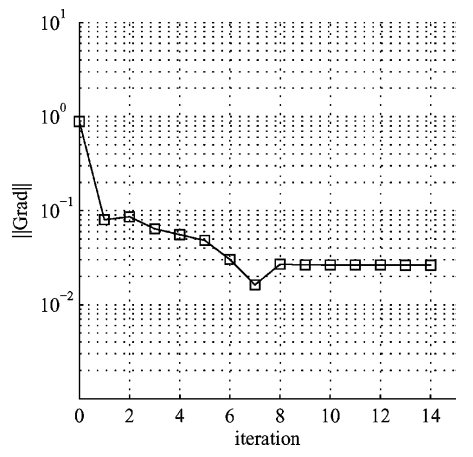

d) Norm of gradient of objective function

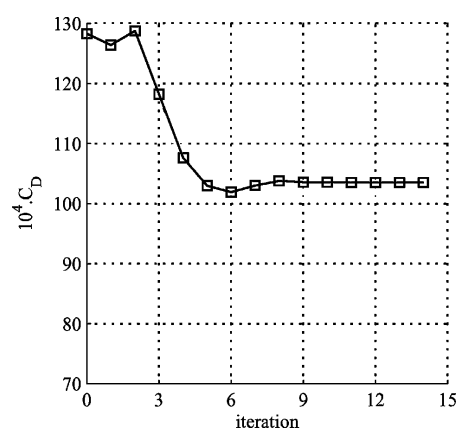

e) Drag

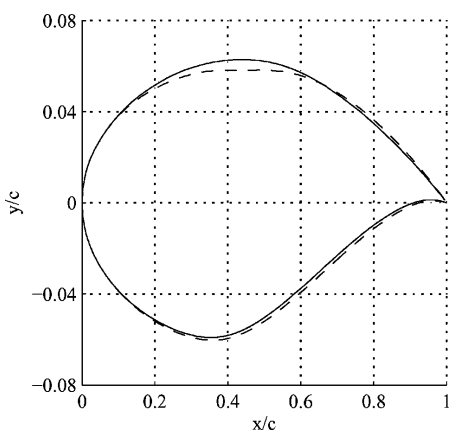

f) Airfoils

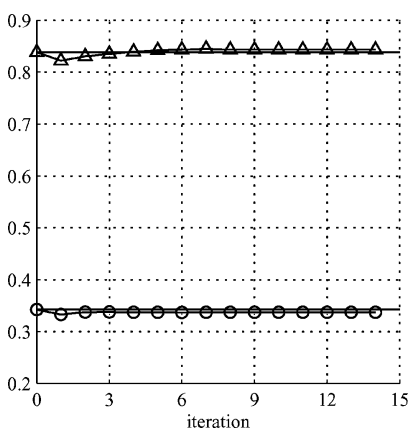

g) Lift and pitch moment

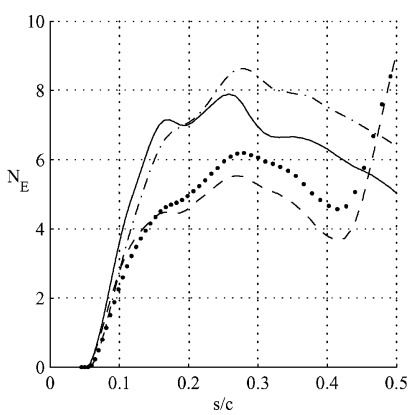

h) Envelope of envelope of $N$-factor curves

Fig. 7 Case T31, comparison between —_, initial and - - --, final design; pressure and EOE of $N$-factor curves involving RANS equations for -.---, initial and $\cdots .$. , final design. 


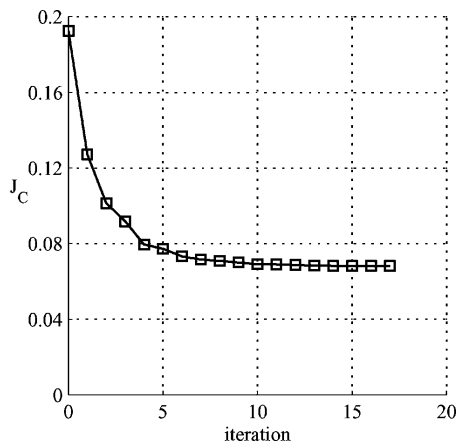

a) Objective function

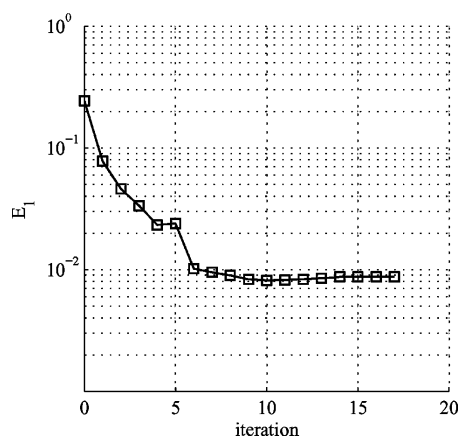

b) Disturbance kinetic energy

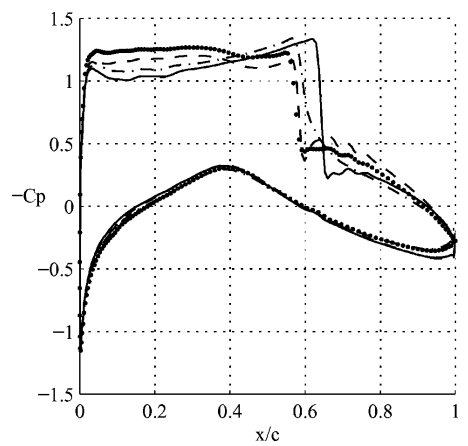

c) Pressure coefficients

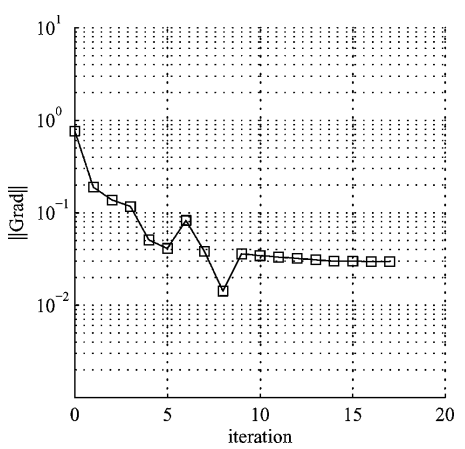

d) Norm of gradient of objective function

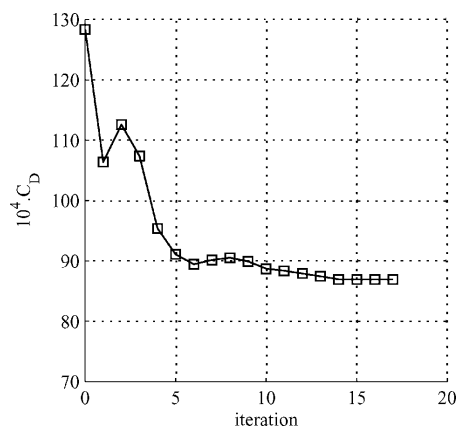

e) Drag

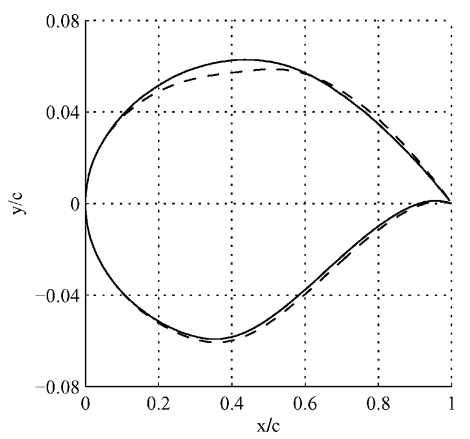

f) Airfoils

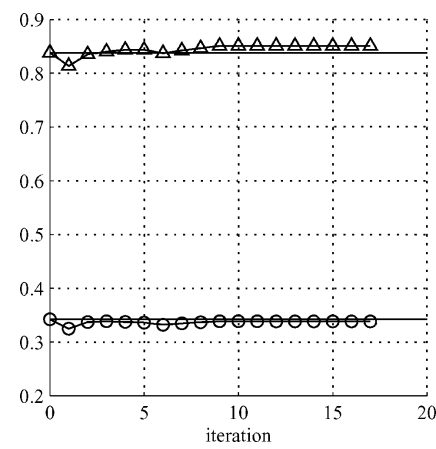

g) Lift and pitch moment

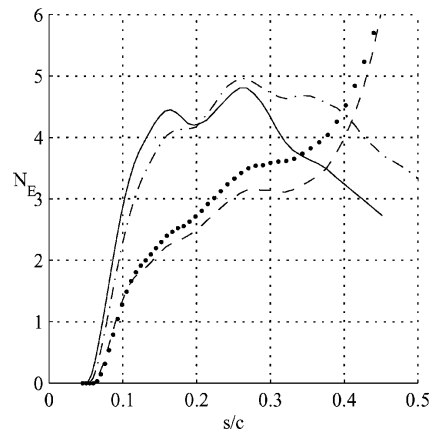

h) Envelope of envelope of $\boldsymbol{N}$-factor curves

Fig. 8 T32, comparison between —_, initial and - - - , final design; pressure and EOE of $N$-factor curves involving RANS equations for -·-_--, initial and $\cdots .$. , final design.

the flow is decelerated. This can be seen in the EOE curve where the value increases rapidly above that of the initial design.

Values of the drag, lift, and pitch-moment coefficients for the initial and optimized designs are summarized in Table 2. The decrease of the viscous drag is somewhat larger than for case T11, but the decrease of the pressure drag is smaller. However, the lift and pitchmoment coefficients are maintained very near to their initial values.

\section{2. $\quad$ Case T32}

Results of case T32 are presented in Fig. 8. The convergence history for the lower Reynolds number case T32 is similar compared to the one found for case T31. Also, here the wave drag experiences an increase during one optimization step, whereas the deviation of lift and pitch-moment coefficients decreases. In comparison with case T12, which has the same initial conditions, the displacement of the shock is smaller, but the pressure distribution in case T32 penalizes changes in the coefficients of lift and pitch moment. A decrease occurs in the $N$-factor values in both results showing the EOE curves of the final design. This can be explained by the change
Table 3 Comparison of aerodynamic coefficients at initial and final design for cases T12 and T32 (9600 m ASL), using Euler and RANS flow analysis

\begin{tabular}{lcccr}
\hline \hline & \multicolumn{3}{c}{ RANS } & \multicolumn{1}{c}{$\begin{array}{c}\text { Euler, } \\
\text { pressure }\end{array}$} \\
\cline { 2 - 4 } Case & Total & Viscous & Pressure & \\
\hline Initial & $2.3 \times 10^{-2}$ & $\begin{array}{c}\text { Coefficient } C_{D} \\
4.8 \times 10^{-3}\end{array}$ & $1.8 \times 10^{-2}$ & $1.3 \times 10^{-2}$ \\
T12 (final) & $1.62 \times 10^{-2}$ & $4.20 \times 10^{-3}$ & $1.20 \times 10^{-3}$ & $7.00 \times 10^{-3}$ \\
T32 (final) & $1.92 \times 10^{-2}$ & $3.72 \times 10^{-3}$ & $1.54 \times 10^{-2}$ & $8.69 \times 10^{-3}$ \\
& & ${\text { Coefficient } C_{L}}^{-3}$ & \\
Initial & $8.4 \times 10^{-1}$ & $-7.9 \times 10^{-5}$ & $8.4 \times 10^{-1}$ & $8.4 \times 10^{-1}$ \\
T12 (final) & $6.87 \times 10^{-1}$ & $-8.00 \times 10^{-5}$ & $6.87 \times 10^{-1}$ & $6.72 \times 10^{-1}$ \\
T32 (final) & $8.68 \times 10^{-1}$ & $-8.3 \times 10^{-5}$ & $8.68 \times 10^{-1}$ & $8.51 \times 10^{-1}$ \\
& & Coefficient $C_{M}$ & & \\
Initial & $3.2 \times 10^{-1}$ & - & - & $3.4 \times 10^{-1}$ \\
T12 (final) & $2.29 \times 10^{-1}$ & - & - & $2.44 \times 10^{-1}$ \\
T32 (final) & $3.21 \times 10^{-1}$ & - & - & $3.38 \times 10^{-1}$ \\
\hline \hline
\end{tabular}


in pressure gradient from adverse to zero or favorable in a large part of the region where the disturbances are amplified.

Values of the drag, lift, and pitch-moment coefficients for the initial and optimized designs are summarized in Table 3 . The decrease of the viscous drag is larger than for case T12, and the decrease of the pressure drag is smaller than for case T12, but the lift and the pitchmoment coefficients are maintained closed to their initial values.

\section{Summary}

An adjoint method has been developed and applied to perform shape optimization with the aim of transition delay and, thus, a decrease of the viscous drag. The location of laminar-turbulent transition is analyzed using linear stability theory, in which perturbations with infinitesimal amplitude are superimposed on the laminar mean flow. It is then assumed that transition will occur at the location where the total amplification of disturbances, with respect to the first streamwise position where the disturbance starts to grow, attains an empirically determined value whose logarithm is generally denoted by $N$. The inviscid flow is obtained by solving the Euler equations for compressible flows, and the viscous mean flow is obtained from the solution of the BLE for compressible flows on infinite swept wings. The evolution of convectively unstable disturbances is analyzed using the linear PSE. It has been shown that the gradient of the objective function with respect to the design variables can be evaluated from the solution of adjoint of the Euler, BLE, and PSE. Using the adjoint equations, as opposed to other perturbation techniques, constitutes an efficient way to evaluate functional gradients when the number of design variables is large compared to the number of objective functions.

In the present approach, an iterative gradient-based optimization procedure is used, and tests are carried out for transonic flows, with Reynolds numbers of $6.5 \times 10^{6}$ and $1.7 \times 10^{7}$, around the RAE 2822 airfoil. One objective function is formulated to produce a reduction of the disturbance kinetic energy. A second one is formulated to simultaneously reduce the wave drag and the disturbance kinetic energy while maintaining lift and pitch-moment coefficients near their values at initial design. The parameterization of the shape is formulated to generate smooth shapes and to satisfy linear constraints; here the volume, the position of the trailing edge, and a part of the shape around the leading edge are kept constant.

The work presented here is an ongoing project and current efforts are being made to improve the accuracy of the gradients and to include additional physical modeling, for example, to account for the occurrence of separated flows. From the point of view of the grid resolution there is a tradeoff between gradient accuracy and computational efficiency. Coarser grids penalize the accuracy of the sensitivities based on the PDEs (adjoint BLE and adjoint PSE), whereas finer grids penalize the cost of the Euler flow computation. A possible solution is to use interpolation techniques to couple the state equations (Euler and BLE), so that these could be discretized using different resolutions at the surface of the airfoil.

We do not consider the coupling between the pressure distribution and the thickness of the boundary layer, so-called viscous-inviscid interaction. However, the influence of this approximation is quite limited because it has been shown that the reduction of the $N$ factor values, obtained by optimization, is also observed by solving the RANS equations instead of the Euler equations to provide the pressure distribution for the boundary-layer analysis. Furthermore, the strategy used to obtain the gradient by an adjoint approach would remain unchanged if using the RANS equations instead of Euler equations during the optimization process. This development would only require an adjoint of the RANS equations.

As far as the numerical results are concerned, several studies can be performed without major changes to the current implementation. For example, an objective function can be defined such that transition on both sides of the wing is delayed. The objective function can also be the sum of a number of convectively unstable disturbances. Another extension, which can include both objective functions, is to use multiple design points in the optimization. In such a case the objective function is the sum of a chosen cost function at different Mach numbers and/or different disturbances.

\section{Appendix A: Sensitivities of State Equations}

The objective function $J$ in expression (18) depends explicitly on $\tilde{\boldsymbol{Q}}$ and on the (Euler) mesh, represented here by the vector of nodal coordinates $\boldsymbol{X}$. (The nodes on the airfoil are common to the three discretized equations: Euler, BLE, and PSE.) The aim is to minimize $J$, where $\tilde{\boldsymbol{Q}}$ is the solution of the PSE (13) and (14), given here as

$$
\mathcal{A}_{\tilde{Q}}(\tilde{\boldsymbol{Q}}, \overline{\boldsymbol{Q}}, \boldsymbol{X})=\mathbf{0}
$$

defined for given $\boldsymbol{X}$ and $\overline{\boldsymbol{Q}}$. The mean flow $\overline{\boldsymbol{Q}}$ is solution of the BLE (8-11), denoted here

$$
\mathcal{A}_{\bar{Q}}(\bar{Q}, \boldsymbol{w}, \boldsymbol{X})=\mathbf{0}
$$

which is defined for a given $\boldsymbol{X}$ and $\boldsymbol{w}$. Finally, the inviscid flow $\boldsymbol{w}$ is the solution of the Euler equations (1) and (2), denoted

$$
\mathcal{A}_{w}(\boldsymbol{w}, \boldsymbol{X})=\mathbf{0}
$$

In the presentation of the adjoint problems, it will be convenient to introduce the functions $J_{X}, J_{\bar{Q}}$, and $J_{w}$, defined as the objective function

$$
\begin{array}{ccc}
V_{\tilde{Q}} \times V_{X} & & \mathbb{R} \\
\{\tilde{\boldsymbol{Q}}, \boldsymbol{X}\} & \longrightarrow & J(\tilde{\boldsymbol{Q}}, \boldsymbol{X})
\end{array}
$$

subject to Eq. (A1)

$$
\begin{array}{ccc}
V_{\bar{Q}} \times V_{X} & \mathbb{R} \\
\{\overline{\boldsymbol{Q}}, \boldsymbol{X}\} & \longrightarrow & J_{\bar{Q}}(\overline{\boldsymbol{Q}}, \boldsymbol{X})
\end{array} \quad \equiv J(\tilde{\boldsymbol{Q}}(\overline{\boldsymbol{Q}}, \boldsymbol{X}), \boldsymbol{X})
$$

or, subject to Eqs. (A1) and (A2),

$$
\begin{array}{ccc}
V_{w} \times V_{X} \\
\{\boldsymbol{w}, \boldsymbol{X}\}
\end{array} \longrightarrow \begin{gathered}
\mathbb{R} \\
J_{w}(\boldsymbol{w}, \boldsymbol{X})
\end{gathered} \quad \equiv J_{\bar{Q}}(\overline{\boldsymbol{Q}}(\boldsymbol{w}, \boldsymbol{X}), \boldsymbol{X})
$$

or, subject to Eqs. (A1-A3),

$$
\begin{array}{lll}
V_{X} & \mathbb{R} \\
\boldsymbol{X} & \longrightarrow & J_{X}(\boldsymbol{X})
\end{array} \equiv J_{w}(\boldsymbol{w}(\boldsymbol{X}), \boldsymbol{X})
$$

These are just function $J$ in which various intermediate quantities are regarded as independent variables.

Given the function $J_{X}$ of the variable $\boldsymbol{X}$ just defined, it will also be convenient to define $J_{y}$ and $J_{a}$ as

$$
J_{y}(\boldsymbol{y})=J_{X}(\boldsymbol{X}(\boldsymbol{y})), \quad J_{a}(\boldsymbol{a})=J_{y}(\boldsymbol{y}(\boldsymbol{a}))
$$

where $\boldsymbol{X}$ is obtained by Eq. (34) and $\boldsymbol{y}$ is the solution of Eq. (39).

To summarize, the aim is to compute $\nabla J_{a}$, that is, the gradient of $J$ subject to Eqs. (A1-A3), (34), and (39) with respect to the design parameters $\boldsymbol{a}$. In the following text it is assumed that $\tilde{\boldsymbol{Q}} \in V_{\tilde{Q}}$, $\overline{\boldsymbol{Q}} \in V_{\bar{Q}}, \boldsymbol{w} \in V_{w}$, and $\boldsymbol{X} \in V_{X}$, and that $V_{\tilde{Q}}, V_{\bar{Q}}, V_{w}$, and $V_{X}$ are vector spaces equipped with the inner products $\langle\cdot, \cdot\rangle_{\tilde{Q}},\langle\cdot, \cdot\rangle_{\bar{Q}},\langle\cdot, \cdot\rangle_{w}$, and $\langle\cdot, \cdot\rangle_{X}$, respectively. Furthermore, it is assumed that all mappings are differentiable and, for example, $\partial \mathcal{A}_{q} / \partial \tilde{\boldsymbol{Q}}$ denotes linearization with respect to variable $\tilde{\boldsymbol{Q}}$ of the mapping $\mathcal{A}_{\tilde{Q}}$, at the given state $\{\tilde{\boldsymbol{Q}}, \overline{\boldsymbol{Q}}, \boldsymbol{w}, \boldsymbol{X}\}$. The notations $\left(\partial \mathcal{A}_{q} / \partial \tilde{\boldsymbol{Q}}\right)^{-1}$ and $\left(\partial \mathcal{A}_{q} / \partial \tilde{\boldsymbol{Q}}\right)^{*}$ denote the inverse and the adjoint of the linearized mapping $\partial \mathcal{A}_{q} / \partial \tilde{\boldsymbol{Q}}$, respectively. Finally, the notation $\left(\partial \mathcal{A}_{q} / \partial \tilde{\boldsymbol{Q}}\right) \delta \tilde{\boldsymbol{Q}}$ denotes the application of $\partial \mathcal{A}_{q} / \partial \tilde{\boldsymbol{Q}}$ on $\delta \tilde{\boldsymbol{Q}}$.

\section{A. Sensitivity of PSE}

For arbitrary variations $\{\delta \overline{\boldsymbol{Q}}, \delta \boldsymbol{X}\} \in V_{\bar{Q}} \times V_{X}$ of $\{\overline{\boldsymbol{Q}}, \boldsymbol{X}\}$ in the PSE (A1), the first variation of the solution of the PSE is denoted $\delta \tilde{\boldsymbol{Q}} \in V_{\tilde{Q}}$ and is the solution of the sensitivity equations,

$$
\frac{\partial \mathcal{A}_{q}}{\partial \tilde{Q}} \delta \tilde{\boldsymbol{Q}}=-\frac{\partial \mathcal{A}_{q}}{\partial \overline{\boldsymbol{Q}}} \delta \overline{\boldsymbol{Q}}-\frac{\partial \mathcal{A}_{q}}{\partial \boldsymbol{X}} \delta \boldsymbol{X}
$$


Furthermore, for any variations $\{\delta \tilde{\boldsymbol{Q}}, \delta \boldsymbol{X}\}$ in $V_{\tilde{Q}} \times V_{X}$ we define the first variation of the objective function $J$ as

$$
\delta J=\left\langle\frac{\partial J}{\partial \tilde{\boldsymbol{Q}}}, \delta \tilde{\boldsymbol{Q}}\right\rangle_{\tilde{Q}}+\left\langle\frac{\partial J}{\partial \boldsymbol{X}}, \delta \boldsymbol{X}\right\rangle_{X}
$$

In the remainder of this paper, $\delta \tilde{\boldsymbol{Q}}$ is the solution of the sensitivity equations (A5), which yields a new expression for Eq. (A6)

$$
\delta J=\left\langle\frac{\partial J}{\partial \tilde{\boldsymbol{Q}}},\left(\frac{\partial \mathcal{A}_{q}}{\partial \tilde{Q}}\right)^{-1}\left(-\frac{\partial \mathcal{A}_{q}}{\partial \bar{Q}} \delta \overline{\boldsymbol{Q}}-\frac{\partial \mathcal{A}_{q}}{\partial \boldsymbol{X}} \delta \boldsymbol{X}\right)\right\rangle_{\tilde{Q}}+\left\langle\frac{\partial J}{\partial \boldsymbol{X}}, \delta \boldsymbol{X}\right\rangle_{X}
$$

and, for the $\tilde{\boldsymbol{Q}}$ solution of Eq. (A1) and the $\delta \tilde{\boldsymbol{Q}}$ solution of Eq. (A5), the definition of $J_{\bar{Q}}$ given earlier yields

$$
\delta J_{\bar{Q}}=\delta J
$$

The gradient $\left(\nabla J_{\bar{Q}}\right)$ of the functional $J_{\bar{Q}}$ is $\left\{\partial J_{\bar{Q}} / \partial \bar{Q}, \partial J_{\bar{Q}} / \partial X_{-}\right\}$and is a vector of the product space $V_{\bar{Q}} \times V_{X}$ such that for all $\{\delta \bar{Q}, \delta \boldsymbol{X}\}$ in $V_{\bar{Q}} \times V_{X}$ we have

$$
\delta J_{\bar{Q}}=\left\langle\frac{\partial J_{\bar{Q}}}{\partial \overline{\boldsymbol{Q}}}, \delta \overline{\boldsymbol{Q}}\right\rangle_{\bar{Q}}+\left\langle\frac{\partial J_{\bar{Q}}}{\partial \boldsymbol{X}}, \delta \boldsymbol{X}\right\rangle_{X}
$$

Using the definition of the adjoint of the operator $\partial \mathcal{A}_{q} / \partial \tilde{\boldsymbol{Q}}$ in expression (A7) and using Eq. (A8), we obtain

$$
\delta J_{\bar{Q}}=\left\langle\left[\left(\frac{\partial \mathcal{A}_{q}}{\partial \tilde{Q}}\right)^{-1}\right]^{*} \frac{\partial J}{\partial \tilde{\boldsymbol{Q}}},-\frac{\partial \mathcal{A}_{q}}{\partial \bar{Q}} \delta \overline{\boldsymbol{Q}}-\frac{\partial \mathcal{A}_{q}}{\partial \boldsymbol{X}} \delta \boldsymbol{X}\right\rangle_{\tilde{Q}}+\left\langle\frac{\partial J}{\partial \boldsymbol{X}}, \delta \boldsymbol{X}\right\rangle_{X}
$$

which is in turn rewritten using the definition of the adjoint of $\partial \mathcal{A}_{q} / \partial \tilde{\boldsymbol{Q}}$ and $\partial \mathcal{A}_{q} / \partial \boldsymbol{X}$, respectively, as

$$
\begin{aligned}
\delta J_{\bar{Q}} & =-\left\langle\left(\frac{\partial \mathcal{A}_{q}}{\partial \bar{Q}}\right)^{*}\left[\left(\frac{\partial \mathcal{A}_{q}}{\partial \tilde{\boldsymbol{Q}}}\right)^{-1}\right]^{*} \frac{\partial J}{\partial \tilde{\boldsymbol{Q}}}, \delta \overline{\boldsymbol{Q}}\right\rangle_{\bar{Q}} \\
& -\left\langle\left(\frac{\partial \mathcal{A}_{q}}{\partial \boldsymbol{X}}\right)^{*}\left[\left(\frac{\partial \mathcal{A}_{q}}{\partial \tilde{Q}}\right)^{-1}\right]^{*} \frac{\partial J}{\partial \tilde{\boldsymbol{Q}}}, \delta \boldsymbol{X}\right\rangle_{X}+\left\langle\frac{\partial J}{\partial \boldsymbol{X}}, \delta \boldsymbol{X}\right\rangle_{X}
\end{aligned}
$$

Therefore, by introducing the adjoint state solution $\tilde{Q}^{*}$ of the system

$$
\left(\frac{\partial \mathcal{A}_{q}}{\partial \tilde{Q}}\right)^{*} \tilde{\boldsymbol{Q}}^{*}=\frac{\partial J}{\partial \tilde{\boldsymbol{Q}}}
$$

we obtain

$$
\frac{\partial J_{\bar{Q}}}{\partial \overline{\boldsymbol{Q}}}=-\left(\frac{\partial \mathcal{A}_{q}}{\partial \bar{Q}}\right)^{*} \tilde{\boldsymbol{Q}}^{*}, \quad \frac{\partial J_{\bar{Q}}}{\partial \boldsymbol{X}}=\frac{\partial J}{\partial \boldsymbol{X}}-\left(\frac{\partial \mathcal{A}_{q}}{\partial \boldsymbol{X}}\right)^{*} \tilde{\boldsymbol{Q}}^{*}
$$

The cost for obtaining the gradient of $J_{\bar{Q}}$ is reduced to one solution of the system (A12) and two matrix-vector products as shown in Eq. (A13).

\section{B. Sensitivity of BLE}

For arbitrary variations $\{\delta \boldsymbol{w}, \delta \boldsymbol{X}\} \in V_{w} \times V_{X}$ of $\{\boldsymbol{w}, \boldsymbol{X}\}$ in the BLE (A2), the first variation of the solution of the BLE is denoted $\delta \overline{\boldsymbol{Q}} \in V_{\bar{Q}}$ and is the solution of the sensitivity equations

$$
\frac{\partial \mathcal{A}_{Q}}{\partial \overline{\boldsymbol{Q}}} \delta \overline{\boldsymbol{Q}}=-\frac{\partial \mathcal{A}_{Q}}{\partial \boldsymbol{w}} \delta \boldsymbol{w}-\frac{\partial \mathcal{A}_{Q}}{\partial \boldsymbol{X}} \delta \boldsymbol{X}
$$

Furthermore, from the definition (A9) and the expression of the gradient (A13) for arbitrary variations $\{\delta \overline{\boldsymbol{Q}}, \delta \boldsymbol{X}\}$ in $V_{\bar{Q}} \times V_{X}$, the variation $\delta J_{\bar{Q}}$ is

$$
\delta J_{\bar{Q}}=\left\langle-\left(\frac{\partial \mathcal{A}_{q}}{\partial \overline{\boldsymbol{Q}}}\right)^{*} \tilde{\boldsymbol{Q}}^{*}, \delta \overline{\boldsymbol{Q}}\right\rangle_{\bar{Q}}+\left\langle\frac{\partial J}{\partial \boldsymbol{X}}-\left(\frac{\partial \mathcal{A}_{q}}{\partial \boldsymbol{X}}\right)^{*} \tilde{\boldsymbol{Q}}^{*}, \delta \boldsymbol{X}\right\rangle_{X}
$$

In the following text, $\delta \bar{Q}$ is the solution of the sensitivity equation (A14). The variation $\delta J_{\bar{Q}}$ is expressed, making use of Eqs. (A15) and (A14) as

$$
\begin{aligned}
\delta J_{\bar{Q}} & =\left\langle\left(\frac{\partial \mathcal{A}_{q}}{\partial \overline{\boldsymbol{Q}}}\right)^{*} \tilde{\boldsymbol{Q}}^{*},\left(\frac{\partial \mathcal{A}_{Q}}{\partial \overline{\boldsymbol{Q}}}\right)^{-1}\left(\frac{\partial \mathcal{A}_{Q}}{\partial \boldsymbol{w}} \delta \boldsymbol{w}+\frac{\partial \mathcal{A}_{Q}}{\partial \boldsymbol{X}} \delta \boldsymbol{X}\right)\right\rangle_{\bar{Q}} \\
& +\left\langle\frac{\partial J}{\partial \boldsymbol{X}}-\left(\frac{\partial \mathcal{A}_{q}}{\partial \boldsymbol{X}}\right)^{*} \tilde{\boldsymbol{Q}}^{*}, \delta \boldsymbol{X}\right\rangle_{X}
\end{aligned}
$$

and, for the solution $\overline{\boldsymbol{Q}}$ of Eq. (A2) and the solution $\delta \overline{\boldsymbol{Q}}$ of Eq. (A14), the definition of $J_{w}$ yields

$$
\delta J_{w}=\delta J_{\bar{Q}}
$$

The gradient of $J_{w}$ is the vector $\left\{\partial J_{w} / \partial \boldsymbol{w}, \partial J_{w} / \partial \boldsymbol{X}\right\}$ in the product space $V_{w} \times V_{X}$ such that for all $\{\delta \boldsymbol{w}, \delta \boldsymbol{X}\}$ in $V_{w} \times V_{X}$ we have

$$
\delta J_{w}=\left\langle\frac{\partial J_{w}}{\partial \boldsymbol{w}}, \delta \boldsymbol{w}\right\rangle_{w}+\left\langle\frac{\partial J_{w}}{\partial \boldsymbol{X}}, \delta \boldsymbol{X}\right\rangle_{X}
$$

When the adjoint of the inverse linearized BLE operator $\left(\partial \mathcal{A}_{Q} / \partial \overline{\boldsymbol{Q}}\right)^{-1}$ is used in Eq. (A16), $\delta J_{w}$ [Eq. (A17)] is expressed as

$$
\begin{aligned}
\delta J_{w} & =\left\langle\left[\left(\frac{\partial \mathcal{A}_{Q}}{\partial \overline{\boldsymbol{Q}}}\right)^{-1}\right]^{*}\left(\frac{\partial \mathcal{A}_{q}}{\partial \overline{\boldsymbol{Q}}}\right)^{*} \tilde{\boldsymbol{Q}}^{*}, \frac{\partial \mathcal{A}_{Q}}{\partial \boldsymbol{w}} \delta \boldsymbol{w}+\frac{\partial \mathcal{A}_{Q}}{\partial \boldsymbol{X}} \delta \boldsymbol{X}\right\rangle_{\bar{Q}} \\
& +\left\langle\frac{\partial J}{\partial \boldsymbol{X}}-\left(\frac{\partial \mathcal{A}_{q}}{\partial \boldsymbol{X}}\right)^{*} \tilde{\boldsymbol{Q}}^{*}, \delta \boldsymbol{X}\right\rangle_{X}
\end{aligned}
$$

Using the adjoints of $\partial \mathcal{A}_{Q} / \partial \boldsymbol{w}$ and $\partial \mathcal{A}_{Q} / \partial \boldsymbol{X}$ enables us to rewrite relation (A19) as

$$
\begin{aligned}
\delta J_{w} & =\left\langle\left(\frac{\partial \mathcal{A}_{Q}}{\partial \boldsymbol{w}}\right)^{*}\left[\left(\frac{\partial \mathcal{A}_{Q}}{\partial \overline{\boldsymbol{Q}}}\right)^{-1}\right]^{*}\left(\frac{\partial \mathcal{A}_{q}}{\partial \overline{\boldsymbol{Q}}}\right)^{*} \tilde{\boldsymbol{Q}}^{*}, \delta \boldsymbol{w}\right\rangle_{w} \\
& +\left\langle\left(\frac{\partial \mathcal{A}_{Q}}{\partial \boldsymbol{X}}\right)^{*}\left[\left(\frac{\partial \mathcal{A}_{Q}}{\partial \overline{\boldsymbol{Q}}}\right)^{-1}\right]^{*}\left(\frac{\partial \mathcal{A}_{q}}{\partial \overline{\boldsymbol{Q}}}\right)^{*} \tilde{\boldsymbol{Q}}^{*}, \delta \boldsymbol{X}\right\rangle_{X} \\
& +\left\langle\frac{\partial J}{\partial \boldsymbol{X}}-\left(\frac{\partial \mathcal{A}_{q}}{\partial \boldsymbol{X}}\right)^{*} \tilde{\boldsymbol{Q}}^{*}, \delta \boldsymbol{X}\right\rangle_{X}
\end{aligned}
$$

This suggests, as earlier, defining an adjoint state $\overline{\boldsymbol{Q}}^{*}$ as

$$
\left(\frac{\partial \mathcal{A}_{Q}}{\partial \overline{\boldsymbol{Q}}}\right)^{*} \overline{\boldsymbol{Q}}^{*}=\left(\frac{\partial \mathcal{A}_{q}}{\partial \overline{\boldsymbol{Q}}}\right)^{*} \tilde{\boldsymbol{Q}}^{*}
$$

Setting $\overline{\boldsymbol{Q}}^{*}$ in Eq. (A20) and identifying the new expression with Eq. (A18), we obtain

$$
\begin{gathered}
\frac{\partial J_{w}}{\partial \boldsymbol{w}}=\left(\frac{\partial \mathcal{A}_{Q}}{\partial \boldsymbol{w}}\right)^{*} \overline{\boldsymbol{Q}}^{*} \\
\frac{\partial J_{w}}{\partial \boldsymbol{X}}=\frac{\partial J}{\partial \boldsymbol{X}}-\left(\frac{\partial \mathcal{A}_{q}}{\partial \boldsymbol{X}}\right)^{*} \tilde{\boldsymbol{Q}}^{*}+\left(\frac{\partial \mathcal{A}_{Q}}{\partial \boldsymbol{X}}\right)^{*} \overline{\boldsymbol{Q}}^{*}
\end{gathered}
$$

The use of adjoint equations limits the cost for obtaining the gradient of $J_{w}$ to solving the systems (A12) and (A21), as well as four matrixvector products: one to assemble the right-hand side of the adjoint system (A21) and three to obtain the final expression (A22).

\section{Sensitivity of the Euler equations}

For arbitrary variations $\delta \boldsymbol{X} \in V_{X}$ of $\boldsymbol{X}$ in the Euler equation (A3), the first variation of solution of the Euler equation is denoted $\delta \boldsymbol{w} \in V_{w}$ and is solution of the sensitivity equation

$$
\frac{\partial \mathcal{A}_{w}}{\partial \boldsymbol{w}} \delta \boldsymbol{w}=-\frac{\partial \mathcal{A}_{w}}{\partial \boldsymbol{X}} \delta \boldsymbol{X}
$$


Furthermore, for arbitrary variations $\{\delta \boldsymbol{w}, \delta \boldsymbol{X}\}$ in $V_{w} \times V_{X}$, the first variation of the functional $J_{w}$ is expressed, from the gradient (A22)

$\delta J_{w}=\left\langle\left(\frac{\partial \mathcal{A}_{Q}}{\partial \boldsymbol{w}}\right)^{*} \overline{\boldsymbol{Q}}^{*}, \delta \boldsymbol{w}\right\rangle_{w}+\left\langle\frac{\partial J}{\partial \boldsymbol{X}}-\left(\frac{\partial \mathcal{A}_{q}}{\partial \boldsymbol{X}}\right)^{*} \tilde{\boldsymbol{Q}}^{*}+\left(\frac{\partial \mathcal{A}_{Q}}{\partial \boldsymbol{X}}\right)^{*} \overline{\boldsymbol{Q}}^{*}, \delta \boldsymbol{X}\right\rangle_{X}$

In the following text, $\delta \boldsymbol{w}$ is the solution of the sensitivity equation (A23), which enables us to rewrite expression (A24) as

$$
\begin{aligned}
\delta J_{w} & =\left\langle\left(\frac{\partial \mathcal{A}_{Q}}{\partial \boldsymbol{w}}\right)^{*} \overline{\boldsymbol{Q}}^{*},-\left(\frac{\partial \mathcal{A}_{w}}{\partial \boldsymbol{w}}\right)^{-1} \frac{\partial \mathcal{A}_{w}}{\partial \boldsymbol{X}} \delta \boldsymbol{X}\right\rangle_{w} \\
& +\left\langle\frac{\partial J}{\partial \boldsymbol{X}}-\left(\frac{\partial \mathcal{A}_{q}}{\partial \boldsymbol{X}}\right)^{*} \tilde{\boldsymbol{Q}}^{*}+\left(\frac{\partial \mathcal{A}_{q}}{\partial \boldsymbol{X}}\right)^{*} \overline{\boldsymbol{Q}}^{*}, \delta \boldsymbol{X}\right\rangle_{X}
\end{aligned}
$$

and, for solution $\boldsymbol{w}$ of Eq. (A3) and solution $\delta \boldsymbol{w}$ of Eq. (A23), the definition of $J_{X}$ yields

$$
\delta J_{X}=\delta J_{w}
$$

The gradient of $J_{X}$ is the vector $\nabla J_{X}$ in the space $V_{X}$ such that for all $\delta \boldsymbol{X}$ in $V_{X}$ we have

$$
\delta J_{X}=\left\langle\nabla J_{X}, \delta \boldsymbol{X}\right\rangle_{X}
$$

The adjoint of the linearized Euler operator is used in Eq. (A25) to express $\delta J_{X}$ [Eq. (A26)] as

$$
\begin{aligned}
\delta J_{X} & =\left\langle\left[\left(\frac{\partial \mathcal{A}_{w}}{\partial \boldsymbol{w}}\right)^{-1}\right]^{*}\left(\frac{\partial \mathcal{A}_{Q}}{\partial \boldsymbol{w}}\right)^{*} \overline{\boldsymbol{Q}}^{*},-\frac{\partial \mathcal{A}_{w}}{\partial \boldsymbol{X}} \delta \boldsymbol{X}\right\rangle_{w} \\
& +\left\langle\frac{\partial J}{\partial \boldsymbol{X}}-\left(\frac{\partial \mathcal{A}_{q}}{\partial \boldsymbol{X}}\right)^{*} \tilde{\boldsymbol{Q}}^{*}+\left(\frac{\partial \mathcal{A}_{Q}}{\partial \boldsymbol{X}}\right)^{*} \overline{\boldsymbol{Q}}^{*}, \delta \boldsymbol{X}\right\rangle_{X}
\end{aligned}
$$

The adjoint instead of the linear operator $\partial \mathcal{A}_{w} / \partial \boldsymbol{X}$ is used in Eq. (A28) and leads to

$$
\begin{aligned}
\delta J_{X} & =\left\langle\frac{\partial J}{\partial \boldsymbol{X}}-\left(\frac{\partial \mathcal{A}_{q}}{\partial \boldsymbol{X}}\right)^{*} \tilde{\boldsymbol{Q}}^{*}+\left(\frac{\partial \mathcal{A}_{Q}}{\partial \boldsymbol{X}}\right)^{*} \overline{\boldsymbol{Q}}^{*}, \delta \boldsymbol{X}\right\rangle_{X} \\
& -\left\langle\left(\frac{\partial \mathcal{A}_{w}}{\partial \boldsymbol{X}}\right)^{*}\left[\left(\frac{\partial \mathcal{A}_{w}}{\partial \boldsymbol{w}}\right)^{-1}\right]^{*}\left(\frac{\partial \mathcal{A}_{Q}}{\partial \boldsymbol{w}}\right)^{*} \overline{\boldsymbol{Q}}^{*}, \delta \boldsymbol{X}\right\rangle_{X}
\end{aligned}
$$

The method of adjoint is again applied as we define an adjoint state $\boldsymbol{w}^{*}$, here the solution of the system

$$
\left(\frac{\partial \mathcal{A}_{w}}{\partial \boldsymbol{w}}\right)^{*} \boldsymbol{w}^{*}=\left(\frac{\partial \mathcal{A}_{Q}}{\partial \boldsymbol{w}}\right)^{*} \overline{\boldsymbol{Q}}^{*}
$$

which enables us to give expression for the gradient (A27),

$$
\nabla J_{X}=\frac{\partial J}{\partial \boldsymbol{X}}-\left(\frac{\partial \mathcal{A}_{q}}{\partial \boldsymbol{X}}\right)^{*} \tilde{\boldsymbol{Q}}^{*}+\left(\frac{\partial \mathcal{A}_{Q}}{\partial \boldsymbol{X}}\right)^{*} \overline{\boldsymbol{Q}}^{*}-\left(\frac{\partial \mathcal{A}_{w}}{\partial \boldsymbol{X}}\right)^{*} \boldsymbol{w}^{*}
$$

The total cost of this gradient evaluation is three adjoint systems (A12), (A21), and (A30) and five matrix-vector products: two for the assembly of the right-hand sides of the systems (A21) and (A30) and three for the final expression (A31).

\section{Appendix B: Matrix Coefficients}

Coefficients of the matrices used in expression (21) are

$$
\begin{aligned}
& a 1(1,4)=\bar{\rho}, \quad a 1(2,4)=\bar{\rho} \bar{U}, \quad a 1(4,1)=-\bar{U}^{2} \\
& a 1(4,2)=-2 \bar{\rho} \bar{U}, \quad a 2(1,4)=\bar{\rho} \\
& a 2(2,4)=\bar{\rho} \bar{U}-i \alpha \frac{\bar{\mu}}{\operatorname{Re}}\left(\frac{\lambda}{\bar{\mu}}+1\right)
\end{aligned}
$$

$a 2(4,2)=-\bar{\rho} \bar{U} 2+i \alpha \frac{\bar{\mu}}{R e}$

$a 3(1,1)=i \alpha \bar{U}+D_{1}(\bar{U}), \quad a 3(1,2)=D_{1}(\bar{\rho})+\bar{\rho} i \alpha$

$a 3(1,4)=D_{3}(\bar{\rho})+\bar{\rho} m_{13}$

$a 3(2,1)=D_{1}(\bar{U}) \bar{U}+\frac{1}{\gamma M^{2}}\left(D_{1}(\bar{T})+i \alpha \bar{T}\right)$

$a 3(2,2)=\bar{\rho}\left[D_{1}(\bar{U})+i \alpha \bar{U}\right]+2 \alpha^{2} \frac{\bar{\mu}}{\operatorname{Re}}\left(\frac{\lambda}{\bar{\mu}}+2\right)$

$a 3(2,3)=\alpha \beta \frac{\bar{\mu}}{R e}\left(\frac{\lambda}{\bar{\mu}}+1\right)$

$a 3(2,4)=\bar{U} D_{3}(\bar{\rho})+\bar{\rho}\left[D_{3}(\bar{U})+\bar{U} m_{13}\right]-\frac{i \alpha}{\operatorname{Re}} \frac{\mathrm{d} \bar{\mu}}{\mathrm{d} \bar{T}} D_{3}(\bar{T})$

$a 3(2,5)=\frac{1}{\gamma M^{2}}\left[D_{1}(\bar{\rho})+i \alpha \bar{\rho}\right]$

$a 3(3,1)=D_{1}(\bar{V}) \bar{U}$

$a 3(3,2)=D_{1}(\bar{V}) \bar{\rho}+\alpha \beta \frac{\bar{\mu}}{\operatorname{Re}}\left(\frac{\lambda}{\bar{\mu}}+1\right)$

$a 3(3,3)=i \alpha \bar{\rho} \bar{U}+2 \frac{\bar{\mu}}{R e} \alpha^{2}$

$a 3(4,1)=-2 D_{3}(\bar{U}) \bar{U}-\bar{U}^{2} m_{13}+\frac{i \alpha}{\bar{\rho}} \frac{\bar{\mu}}{R e}\left(\frac{\lambda}{\bar{\mu}}+2\right) D_{3}(\bar{U})$

$a 3(4,2)=-2 D_{3}(\bar{\rho} \bar{U})-\frac{i \alpha}{R e} \frac{\mathrm{d} \bar{\mu}}{\mathrm{d} \bar{T}} \frac{\lambda}{\bar{\mu}} D_{3}(\bar{T})-\bar{\rho} \bar{U} 2 m_{13}$

$$
+\frac{i \alpha}{\bar{\rho}} \frac{\bar{\mu}}{\operatorname{Re}}\left(\frac{\lambda}{\bar{\mu}}+2\right) D_{3}(\bar{\rho})
$$

$a 3(4,4)=2 \frac{\bar{\mu}}{\operatorname{Re}} \alpha^{2}+\bar{\rho} \bar{U} i \alpha, \quad a 3(4,5)=-\frac{i \alpha}{\operatorname{Re}} \frac{\mathrm{d} \bar{\mu}}{\mathrm{d} \bar{T}} D_{3}(\bar{U})$

$a 3(5,1)=\frac{(\gamma-1)}{\gamma}\left[\bar{U} \bar{T} i \alpha+\bar{U} D_{1}(\bar{T})\right]-\bar{c}_{p} \bar{U} D_{1}(\bar{T})$

$a 3(5,2)=(\gamma-1) M^{2} D_{1}(\bar{P})-\bar{\rho} \bar{c}_{p} D_{1}(\bar{T})$

$a 3(5,4)=(\gamma-1) M^{2} D_{3}(\bar{U}) 2 i \alpha \frac{\bar{\mu}}{R e}$

$a 3(5,5)=\frac{(\gamma-1)}{\gamma}\left[\bar{U} D_{1}(\bar{\rho})+\bar{U} \bar{\rho} i \alpha\right]$

$$
-\bar{U} \bar{\rho}\left[\bar{c}_{p} i \alpha+\frac{\mathrm{d} \bar{c}_{p}}{\mathrm{~d} \bar{T}} D_{1}(\bar{T})\right]-\frac{2}{\operatorname{RePr}} \bar{\kappa} \alpha^{2}
$$

$a 4(1,1)=\bar{U}, \quad a 4(1,2)=\bar{\rho}, \quad a 4(2,1)=\frac{\bar{T}}{\gamma M^{2}}$

$a 4(2,2)=\bar{\rho} \bar{U}, \quad a 4(2,5)=\frac{\bar{\rho}}{\gamma M^{2}}, \quad a 4(3,3)=\bar{\rho} \bar{U}$

$a 4(4,4)=\bar{\rho} \bar{U}, \quad a 4(5,1)=\bar{U} \bar{T} \frac{(\gamma-1)}{\gamma}$

$a 4(5,5)=\bar{U} \bar{\rho}\left[\frac{(\gamma-1)}{\gamma}-\bar{c}_{p}\right]$ 


\section{Acknowledgments}

The financial support of European Union project Application of Hybrid Laminar Flow Technology on Transport (ALTTA) Contract G4RD-CT-2000-00143 is gratefully acknowledged. This research has received support from the AEROSHAPE Project funded by the European Commission, DG Research, under the GROWTH initiative (Project Reference: GRD1-1999-10752).

\section{References}

${ }^{1}$ Joslin, R., "Overview of Laminar Flow Control," NASA TR 1998-208705, Oct. 1998

${ }^{2}$ Jameson, A., "Optimum Aerodynamic Design Using CFD and Control Theory," AIAA Paper 95-1729, June 1995.

${ }^{3}$ Nemec, M., and Zingg, D., "Towards Efficient Aerodynamic Shape Optimization Based on the Navier-Stokes Equations," AIAA Paper 2001-2532, June 2001.

${ }^{4}$ van Ingen, J. L., "A Suggested Semiempirical Method for the Calculation of the Boundary Layer Transition region," Dept. of Aeronautical Engineering, TR VTH-74, Univ. of Delft, Delft, The Netherlands, 1956.

${ }^{5}$ Smith, A. M. O., and Gamberoni, N., "Transition, Pressure Gradient and Stability Theory," Douglas Aircraft Co., TR ES26388, 1956.

${ }^{6}$ Arnal, D., "Boundary Layer Transition: Predictions Based on Linear Theory," Special Course on Progress in Transition Modelling, AGARD-R793, 1993, pp. 2-1-2-63.

${ }^{7}$ Green, B. E., Whitesides, J. L., Campbell, R. L., and Mineck, R. E., "A Method for the Constrained Design of Natural Laminar Flow Airfoils," Journal of Aircraft, Vol. 34, No. 6, 1997, pp. 706-712.

${ }^{8}$ Manning, V. M., and Kroo, I. M., "Multidisciplinary Optimization of a Natural Laminar Flow Supersonic Aircraft," AIAA Paper 99-3102, JuneJuly 1999

${ }^{9}$ Lions, J., Optimal Control of Systems Governed by Partial Differential Equations, Springer-Verlag, New York, 1971.

${ }^{10}$ Pironneau, O., "On Optimal Profiles in Stokes Flow," Journal of Fluid Mechanics, Vol. 59, 1973, pp. 117-128.

${ }^{11}$ Jameson, A., "Aerodynamic Design via Control Theory," Journal of Scientific Computing, Vol. 3, No. 3, 1988, pp. 233-260.

${ }^{12}$ Anderson, W., and Bonhaus, D., "Airfoil Design on Unstructured Grids for Turbulent Flows," AIAA Journal, Vol. 37, No. 2, 1999, pp. 185-191.

${ }^{13}$ Baysal, O., and Ghayour, K., "Continuous Adjoint Sensitivities for Optimization with General Cost Functionals on Unstructured Meshes," AIAA Journal, Vol. 39, No. 1, 2001, pp. 48-55.

${ }^{14}$ Burgreen, G., Baysal, O., and Eleshaky, M., "Improving the Efficiency of Aerodynamic Shape Optimization," AIAA Journal, Vol. 32, No. 1, 1994, pp. 69-76.

${ }^{15}$ Elliot, J., "Aerodynamic Based on the Euler and Navier-Stokes Equations Using Unstructured Grids," Ph.D. Dissertation, Dept. of Aeronautics and Astronautics, Massachusetts Inst. of Technology, Cambridge, MA, May 1998.

${ }^{16}$ Enoksson, O., "Shape Optimization in Compressible Inviscid Flow," Licenciate Thesis LiU-TEK-LIC-2000:31, Dept. of Mathematics, Inst. of Technology, Linköpings Univ., May 2000.

${ }^{17}$ Jameson, A., Pierce, N., and Martinelly, L., "Optimum Aerodynamic Design Using the Navier-Stokes Equations," AIAA Paper 97-0101, Jan. 1997.

${ }^{18}$ Mohammadi, B., "A New Optimal Shape Procedure for Inviscid and Viscous Turbulent Flows," International Journal for Numerical Methods in Fluids, Vol. 25, No. 2, 1997, pp. 183-203.

${ }^{19}$ Reuther, J. J., Jameson, A., Alonso, J. J., Rimlinger, M. J., and Saunders, D., "Constrained Multipoint Aerodynamic Shape Optimization Using an Adjoint Formulation and Parallel Computers, Part 1," Journal of Aircraft, Vol. 36, No. 1, 1999, pp. 51-60.

${ }^{20}$ Soemarwoto, B., "Multi-Point Aerodynamic Design by Optimization," $\mathrm{Ph} . \mathrm{D}$. Dissertation, Faculty of Aerospace Engineering, Delft Univ. of Technology, Delft, The Netherlands, Dec. 1996.

${ }^{21}$ Sung, C., and Kwon, J., "Accurate Aerodynamic Sensitivity Analysis Using Adjoint Equations," AIAA Journal, Vol. 38, No. 2, 2000, pp. 243-250.

${ }^{22}$ Giles, M., and Pierce, N., "An Introduction to the Adjoint Approach to Design," Flow, Turbulence and Control, Vol. 65, Nos. 3-4, 2000, pp. 393-415.

${ }^{23}$ Schubauer, G. B., and Skramstad, H. K., "Laminar-Boundary-Layer
Oscillations and Transition on a Flat Plate,” NACA TN 909, 1948.

${ }^{24}$ Malik, M. R., "Prediction and Control of Transition in Supersonic and Hypersonic Boundary Layers," AIAA Journal, Vol. 27, No. 11, 1989, pp. 1487-1493.

${ }^{25}$ Zurigat, Y. H., Nayfeh, A. H., and Masad, J. A., "Effect of Pressure Gradient on the Stability of Compressible Boundary Layers," AIAA Journal, Vol. 30, No. 9, 1992, pp. 2204-2211.

${ }^{26}$ Lighthill, M. J., "A New Method of Two-Dimensional Aerodynamic Design," Aeronautical Research Council, R\&M 2112, London, 1945.

${ }^{27}$ Pralits, J. O., and Hanifi, A., "Optimization of Steady Suction for Disturbance Control on Infinite Swept Wings," Physics of Fluids, Vol. 15, No. 9, 2003, pp. 2756-2772.

${ }^{28}$ Pralits, J. O., Hanifi, A., and Henningson, D. S., "Adjoint-Based Optimization of Steady Suction for Disturbance Control in Incompressible Flows," Journal of Fluid Mechanics, Vol. 467, 2002, pp. 129-161.

${ }^{29}$ Eliasson, P., "EDGE, A Navier-Stokes Solver, for Unstructured Grids," Swedish Defence Research Agency, TR FOI-R-0298-SE, Stockholm, Nov. 2001.

${ }^{30}$ Barth, T., "Aspects of Unstructured Grids and Finite-Volume Solvers for the Euler and Navier-Stokes Equations," Special Course on Unstructured Methods for Advection Dominated Flows, Rept. 787, AGARD, 1991, pp. 6-1-6-61.

${ }^{31}$ Bertolotti, F. P., Herbert, T., and Spalart, S., "Linear and Nonlinear Stability of the Blasius Boundary Layer," Journal of Fluid Mechanics, Vol. 242, 1992, pp. 441-474.

${ }^{32}$ Malik, M. R., and Balakumar, P., "Nonparallel Stability of Rotating Disk Flow Using PSE," Instability, Transition and Turbulence, edited by M. Y. Hussaini, A. Kumar, and C. L. Streett, Springer-Verlag, Berlin, 1992, pp. $168-180$.

${ }^{33}$ Simen, M., "Local and Non-Local Stability Theory of Spatially Varying Flows," Instability, Transition and Turbulence, edited by M. Hussaini, A. Kumar, and C. Streett, Springer, New York, 1992, pp. 181-201.

${ }^{34}$ Herbert, T., "Parabolized Stability Equations," Annual Review of Fluid Mechanics, Vol. 29, 1997, pp. 245-283.

${ }^{35}$ Pralits, J. O., Airiau, C., Hanifi, A., and Henningson, D. S., "Sensitivity Analysis Using Adjoint Parabolized Stability Equations for Compressible Flows," Flow, Turbulence and Combustion, Vol. 65, No. 3/4, 2000, pp. 321-346.

${ }^{36}$ Hanifi, A., Henningson, D. S., Hein, S., and Bertolotti, F. P., and Simen, M., "Linear Non-local Instability Analysis-The Linear NOLOT Code," Aeronautical Research Inst. of Sweden, FFA-TN 1994-54, 1994.

${ }^{37}$ Airiau, C., Bottaro, A., Walther, S., and Legendre, D., "A Methodology for Optimal Laminar Flow Control: Application to the Damping of TollmienSchlichting Waves in a Boundary Layer," Physics of Fluids, Vol. 15, No. 5, 2003, pp. 1131-1145.

${ }^{38}$ Amoignon, O., “Adjoint-Based Aerodynamic Shape Optimization,” TR IT Licentiate Thesis 2003-012, Dept. of Information Technology, Div. of Scientific Computing, Uppsala Univ., Uppsala, Sweden, Oct. 2003.

${ }^{39}$ Pralits, J. O., "Towards Optimal Design of Vehicles with Low Drag: Applications to Sensitivity Analysis and Optimal Control," Licentiate Thesis KTH/MEK/TR-01/07, Dept. of Mechanics, Royal Inst. of Technology, Stockholm, April 2001.

${ }^{40}$ Amoignon, O., Pralits, J. O., Hanifi, A., Berggren, M., and Henningson, D. S., "Shape Optimization for Delay of Laminar-Turbulent Transition," Aeronautics Div., Swedish Defence Research Agency, TR FOI-R-0919-SE, Stockholm, Oct. 2003.

${ }^{41}$ Frank, P., and Shubin, G., "A Comparison of Optimization-Based Approaches for a Model Computational Aerodynamics Design Problem," Journal of Computational Physics, Vol. 98, No. 1, 1992, pp. 74-89.

${ }^{42}$ Nocedal, J., and Wright, S., Numerical Optimization, Springer Series in Operations Research, Springer, New York, 1999.

${ }^{43}$ Wallin, S., and Johansson, A. V., "An Explicit Algebraic Reynolds Stress Model for Incompressible and Compressible Turbulent Flows," Journal of Fluid Mechanics, Vol. 403, 2000, pp. 89-132.

${ }^{44}$ Cook, P., McDonald, M., and Firmin, M., "Aerofoil RAE 2822Pressure Distributions, and Boundary Layer and Wake Measurements," Experimental Data Base for Computer Program Assessment, AR-138, AGARD, 1979, pp. A6-1-A6-77.

S. Saigal

Associate Editor 